\title{
ON PARAMETRIZING EXCEPTIONAL TANGENT CONES TO PRYM THETA DIVISORS
}

\author{
ROY SMITH AND ROBERT VARLEY
}

\begin{abstract}
The theta divisor of a Jacobian variety is parametrized by a smooth divisor variety via the Abel map, with smooth projective linear fibers. Hence the tangent cone to a Jacobian theta divisor at any singularity is parametrized by an irreducible projective linear family of linear spaces normal to the corresponding fiber. The divisor variety $X$ parametrizing a Prym theta divisor $\Xi$, on the other hand, is singular over any exceptional point. Hence although the fibers of the Abel Prym map are still smooth, the normal cone in $X$ parametrizing the tangent cone of $\Xi$ can have nonlinear fibers.

In this paper we highlight the diverse and interesting structure that these parametrizing maps can have for exceptional tangent cones to Prym theta divisors. We also propose an organizing framework for the various possible cases. As an illustrative example, we compute the case of a Prym variety isomorphic to the intermediate Jacobian of a cubic threefold, where the projectivized tangent cone, the threefold itself, is parametrized by the 2 parameter family of cubic surfaces cut by hyperplanes through a fixed line on the threefold.
\end{abstract}

\section{INTRODUCTION}

Describing a tangent cone to a Jacobian theta divisor has three important aspects:

i) the numerical one of computing the multiplicity at a point in terms of the dimension of sections of the line bundle associated to that point,

ii) the algebraic one of giving determinantal equations for the tangent cone in terms of such sections, and

iii) the geometric one of parametrizing the tangent cone by a linear family of varieties of infinitesimal variations of divisors associated to such sections.

For Jacobian theta divisors, these descriptions were provided by Riemann Ri for i), by Mumford and Kempf [K1, K2, K3 , for ii), and by Andreotti-Mayer [A-M] and Kempf [K1] for iii). In particular, let $C$ be a smooth irreducible curve of genus $g, C^{(g-1)}$ the $(g-1)$-fold symmetric product of the curve parametrizing effective divisors of degree $g-1$, and $\alpha: C^{(g-1)} \rightarrow \mathrm{Pic}^{g-1}(C)=J(C)$ the Abel map sending a divisor $D$ to the associated line bundle $\alpha(D)=\mathcal{O}(D)$. Then $\alpha$ is a birational parametrization of Riemann's theta divisor $\Theta=\alpha\left(C^{(g-1)}\right)$ by a smooth variety, such that for $L \in \Theta$, the inverse image $\alpha^{-1}(L)=|L| \cong \mathbb{P} H^{0}(C, L)$ is the complete linear series defined by the sections of $L$. Then mult $_{L}(\Theta)=h^{0}(C, L)$, and the tangent cone to $\Theta$ at $L$ is parametrized via the derivative $\alpha_{*}$ by the irreducible normal vector bundle to $|L|$ in $C^{(g-1)}$.

Received by the editors August 5, 2013 and, in revised form, October 28, 2014 and May 13, 2015 .

2010 Mathematics Subject Classification. Primary 14H40; Secondary 14K12. 
If we identify the projective tangent space $\mathbb{P} T_{0}(J(C))$ with the canonical embedding space $\mathbb{P} H^{0}(C, K)^{*} \cong \mathbb{P}^{g-1}$ of the (nonhyperelliptic) curve $C$, then both $C$ and the projective tangent cone $\mathbb{P} C_{L}(\Theta)$ are embedded naturally in this copy of $\mathbb{P}^{g-1}$. The cone $\mathbb{P} C_{L}(\Theta)$ is a reduced irreducible rational hypersurface of degree $h^{0}(C, L)$ ruled by the images under $\alpha_{*}$ of the linear spaces normal to $|L|$ in $C^{(g-1)}$.

Under the canonical embedding in $\mathbb{P}^{g-1}$, the space normal to $|L|$ at $D$ in $C^{(g-1)}$ parametrizes the linear subspace of $\mathbb{P} C_{L}(\Theta)$ spanned by the divisor $D$ on $C$. Finally, if $\left\{s_{0}, \ldots, s_{r}\right\}$ is a basis for $H^{0}(C, L)$ and $\left\{t_{0}, \ldots, t_{r}\right\}$ is a basis for $H^{0}(C, K-L)$, then the hypersurface $\mathbb{P} C_{L}(\Theta)$ is defined as a subscheme of $\mathbb{P}^{g-1}$ by the determinantal equation $\operatorname{det}\left[s_{i} t_{j}\right]=0$. In fact, if $C$ is a nonhyperelliptic curve of genus $g \geq 5$, then rank 4 double points are dense in $\operatorname{Sing}(\Theta)$, and in most cases the canonical curve $C$ is precisely the intersection of the associated rank 4 quadric tangent cones, which provides a constructive proof of Torelli's theorem in those cases G.

In the case of Prym theta divisors, the precise multiplicity was determined relatively recently by Casalaina-Martin [CM], and consequent Pfaffian algebraic equations given by Smith and Varley $[\mathrm{S}-\mathrm{V} 6]$. The geometric parametrization is still lacking in a completely precise form; in particular even for double points the problem of the rank of the quadric tangent cone is not well understood. By analogy with the work of Mark Green [G] on constructive Torelli for Jacobians, this has bearing on the constructive Torelli problem for Pryms which, by the recent counterexamples of Izadi and Lange [I-L], seems to be rather complicated and interesting. For generic Prym varieties of dimension at least 7 , Debarre $[\mathrm{D}]$ showed that the étale double cover of curves can be reconstructed from the double points of the Prym theta divisor.

The case of Jacobians is simpler because the divisor variety provides a smooth birational resolution of the theta divisor with smooth scheme theoretic linear fibers. Consequently the tangent cones are always birationally ruled by a linear family of linear spaces of known constant dimension; e.g. a tangent cone to a Jacobian theta divisor $\Theta$ is always an irreducible rational variety. Except in the hyperelliptic case, this resolution of $\Theta$ is even a "small" one and the "equisingular" deformations of the Jacobian theta divisor correspond exactly to deformations of the divisor variety [S-V2, Lemma, p. 251], at least to first order.

For Pryms, the divisor variety is never birational to the theta divisor $\Xi$ and not always smooth. The scheme theoretic fibers of the Abel Prym map are still smooth, and this implies for Pryms with smooth divisor variety, i.e. those with no exceptional singularities on $\Xi$, that every tangent cone is again parametrized, although not birationally, by a linear family of linear spaces of known constant dimension $[\mathrm{S}-\mathrm{V} 3$. In particular the tangent cone at a nonexceptional singularity of a Prym theta divisor is always irreducible and unirational. This leaves open the problem of describing the geometric parametrization in the presence of exceptional singularities on $\Xi$.

Contents of the paper. This paper makes a beginning on the problem by dividing exceptional singularities of Prym theta divisors into 3 types for which the maps parametrizing the tangent cones have characteristically different structures. In other words, an exceptional singularity can have two progressively more special forms, called "very exceptional" and "totally exceptional", that affect the parametrization of the tangent cone. The terms exceptional, very exceptional, and totally exceptional for singularities of a Prym theta divisor are defined in section 2 
below. Such distinctions depend in general on the étale double cover of curves used to construct the Prym variety $P$ and its theta divisor $\Xi$.

To be precise, if $X$ is the divisor variety parametrizing $\Xi$ and $|L| \subset X$ is the preimage linear series over an exceptional singular point $L \in \Xi$, consider the diagram:

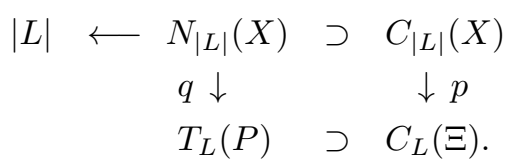

Here, $C_{|L|}(X)$ and $N_{|L|}(X)$ are respectively the normal cone and normal space of $X$ along $|L|$, and similarly $C_{L}(\Xi)$ and $T_{L}(P)$ are the tangent cone to $\Xi$ and the tangent space of $P$ at $L$. The parametrization of the tangent cone is the map $p$, which in the present setting is induced by an ambient map $q$, the Abel Prym differential on Zariski normal vectors. In order to have three disjoint types for an exceptional singular point $L$, we define case (1) as exceptional but not very exceptional, case (2) as very exceptional but not totally exceptional, and case (3) as totally exceptional. In all the cases, there is a unique irreducible component $N_{1}$ of $N_{|L|}(X)$ that dominates $|L| ; N_{1}$ is also an irreducible component of $C_{|L|}(X)$ in cases (1-2), but not in case (3).

The distinction between (1) and (2-3) is whether or not all of $N_{|L|}(X)$ maps into $C_{L}(\Xi)$. (This dichotomy comes from our previous results $[\mathrm{S}-\mathrm{V} 3,5]: q\left(N_{|L|}(X)\right)=$ $T_{L}(P)$ if and only if $L$ is very exceptional.) An easy distinction between cases (2) and (3) is whether or not the component $N_{1}$ maps into $C_{L}(\Xi)$. We show that in case (2) the image of $N_{1}$ has codimension at least 3 in $T_{L}(P)$. It follows that the normal cone $C_{|L|}(X)$ is always reducible in case (2). This result is presented as Theorem 2 and the proof uses properties of Mumford's skew-symmetric pairing $H^{0}(L) \times H^{0}(L) \rightarrow T_{L}^{*}(P)$. Typically, there are glaring differences among the 3 cases, as we will explain below, based just on the structure of the normal cone $C_{|L|}(X)$ and its map to $|L|$.

Our guiding example of a very exceptional singular point has been the unique triple point on the theta divisor of the intermediate Jacobian of a cubic threefold. If $W \subset \mathbb{P}^{4}$ is a smooth cubic threefold, then for a fixed general line $\ell$ in $W$, the sections of $W$ by the planes in $\mathbb{P}^{4}$ through $\ell$ form (residually to $\ell$ ) a conic bundle over a parameter space $\mathbb{P}^{2}$. As is known, the quintic discriminant curve $C \subset \mathbb{P}^{2}$ is smooth and has an étale double cover $\pi: \widetilde{C} \rightarrow C$ that provides a Prym variety realization of the intermediate Jacobian $J(W)$. In Theorem 3 we give a complete description of the Abel Prym parametrization of this well known tangent cone (which projectively is $W$ itself).

We show in this case, contrary to the case of a Jacobian, that if $L=\pi^{*}\left(g_{5}^{2}\right)$ is the line bundle on $\widetilde{C}$ pulled back from $\mathcal{O}(1)$ on the plane quintic $C$, corresponding to the triple point on the Prym theta divisor $\Xi$, then the normal cone to the fiber $|L|$ of the Abel Prym map $X \rightarrow \Xi$ is reducible with two components. Moreover, the only component that dominates the tangent cone to $\Xi$ at $L$ is supported on a proper subvariety of that fiber, in fact on the projective linear subspace $\mathbb{P}\left(\pi^{*} H^{0}\left(g_{5}^{2}\right)\right) \subset|L|$ corresponding to the pulled back sections of the unique "Shokurov" line bundle $M=g_{5}^{2}$ on $C$ that makes $L$ very exceptional. Unlike the case of Jacobians, this parametrization of the projectivized tangent cone $\mathbb{P} C_{L}(\Xi)=W$ is not by a family of linear spaces but by a linear net of cubic surfaces, sections of the threefold $W$ by 
the hyperplanes in $\mathbb{P}^{4}$ passing through the distinguished line $\ell$ in $W$, with parameter space $\left(\mathbb{P}^{2}\right)^{*}$.

The situation for the cubic threefold generalizes somewhat to the case of odd dimensional cubic hypersurfaces that contain in codimension 2 a linear subspace. We also give several other kinds of examples. In general we do not know a detailed description of the parametrizing map for the tangent cone at a very exceptional singular point of a Prym theta divisor. As long as $L \in \Xi$ is not very exceptional, everything about the parametrization of the tangent cone $C_{L}(\Xi)$ can be computed from the Mumford pairing $H^{0}(L) \times H^{0}(L) \rightarrow T_{L}^{*}(P)$ or, relative to a basis for $H^{0}(L)$, a skew-symmetric matrix of linear forms on the tangent space $T_{L}(P)$. In this case the tangent cone is pulled back from a universal Pfaffian cone. We include as Theorem 1 a complete description of the parametrization of $C_{L}(\Xi)$ for any stable double point $L$. We show by example that it is possible for the normal cone $C_{|L|}(X)$ to be reducible even in "case (1)", i.e. for $L$ exceptional but not very exceptional. We describe the geometry of the Pfaffian cone in the space of skew-symmetric matrices and review in general the properties of the linear map from $T_{L}(P)$ to the matrix space. Precisely in the very exceptional case the tangent cone $C_{L}(\Xi)$ is not induced from the universal Pfaffian; but the construction still provides information unless $L$ is totally exceptional, in which case the linear map to matrix space is identically 0 .

Organization of the paper. We recall in section 2 some basic definitions and facts about the theta divisor $\Xi$ of the Prym variety of an étale double cover $\pi$ : $\widetilde{C} \rightarrow C$, the parametrization of $\Xi$ by the restriction of the Abel map of $\widetilde{C}$ to a special divisor variety $X$, and the differential of this Abel Prym map. In section 3 we formulate what the expected typical structure of the parametrization seems to be for each of the basic types $(1,2,3)$ and give a guide as to what is actually proved about the cases and what degeneracies might occur.

Then in section 4, we explain the geometry of Mumford's skew-symmetric matrix of linear forms associated to each point of a Prym theta divisor. We work out all the possibilities for stable double points and summarize the classification in Theorem 1. In section 5, we prove Theorem 2 showing in particular that in "case (2)", of $L$ very exceptional but not totally, the normal cone of the divisor variety along $|L|$ is always reducible and the tangent cone to $\Xi$ at $L$ is parametrized by those components of the normal cone that lie over a proper linear subspace of $|L|$. (The subspace has the form $\pi^{*}|M|+B$ for a uniquely determined line bundle $M$ on $C$ and effective divisor $B$ on $\widetilde{C}$.)

In section 6 , we prove Theorem 3 describing the parametrization of the tangent cone at the (very exceptional) triple point on the theta divisor of the intermediate Jacobian of a cubic threefold, based on a Prym variety realization of the intermediate Jacobian. We also include some other examples to illustrate that certain features of the expected typical structure can indeed occur. In the final section 7 we present an example to show how some of the "expected" structure can break down; in particular a reducible normal cone can occur for an exceptional but not very exceptional singularity.

Throughout this paper we work over the complex numbers $\mathbb{C}$, for simplicity and uniformity. Most of the arguments will still hold unchanged over an algebraically closed field of characteristic $\neq 2$, but we have not checked any additional details or references. 


\section{The PARAMETRIZATION OF A TANGENT CONE}

2.1. The setup. Consider a connected étale double cover $\pi: \tilde{C} \rightarrow C$ of a smooth curve $C$ of genus $g$. We will use $\eta$ to denote the corresponding nontrivial 2-torsion divisor class on $C$ and $\iota$ to denote the involution of $\widetilde{C}$. The associated Prym variety $P_{0}$ is an abelian subvariety of the Jacobian $\widetilde{J}_{0}=\operatorname{Pic}^{0}(\widetilde{C})$ and has the property that the restriction of the principal polarization of $\widetilde{J}_{0}$ is twice a principal polarization for $P_{0}$. We will rely heavily on the alternative realization of $P_{0} \subset \widetilde{J}_{0}$ by principal homogeneous spaces $P \subset \widetilde{J}$ with geometric models for the theta divisors. That is, let $\widetilde{J}=P i c^{2 g-2}(\widetilde{C})$, so that $\widetilde{\Theta}=\left\{L \in \widetilde{J} \mid h^{0}(L)>0\right\}$ is a standard Jacobian theta divisor defining the principal polarization of $\widetilde{J}_{0}$. Then $P=\{L \in \widetilde{J} \mid N m(L) \cong$ $\Omega_{C}$ and $h^{0}(L)$ is even $\}$ and the Prym theta divisor $\Xi$ is defined by $2 \Xi=P \cdot \widetilde{\Theta}$. The basic reference is Mumford's paper [M, section 3, then p. 337 and Prop. (b), p. 342].

2.1.1. Abel Prym map. Let $\tilde{\alpha}: \widetilde{C}^{(2 g-2)} \rightarrow \widetilde{\Theta} \subset \widetilde{J}$ be the Abel map for the smooth curve $\widetilde{C}$ of genus $\tilde{g}=2 g-1$. Thus the smooth variety of effective divisors of degree $\tilde{g}-1=2 g-2$ on $\widetilde{C}$ maps birationally onto the theta divisor in the Jacobian. Then the Prym divisor variety $X$ is the inverse image of $P$, hence also of $\Xi$, under the Abel map, and the induced map $\varphi: X \rightarrow \Xi \subset P$ is the Abel Prym map for the étale double cover $\pi: \widetilde{C} \rightarrow C$. That is,

$$
\begin{aligned}
& X=\left\{q_{1}+\cdots+q_{2 g-2} \in \widetilde{C}^{(2 g-2)} \mid\right. \pi\left(q_{1}\right)+\cdots+\pi\left(q_{2 g-2}\right) \in\left|\Omega_{C}\right| \subset C^{2 g-2} \\
&\text { and } \left.h^{0}\left(\mathcal{O}_{\widetilde{C}}\left(q_{1}+\cdots+q_{2 g-2}\right)\right) \text { is even }\right\},
\end{aligned}
$$

the space of effective divisors of degree $2 g-2$ on $\widetilde{C}$ whose norm on $C$ is in the canonical linear series of $C$ and whose complete linear series on $\widetilde{C}$ is of odd dimension. Then we denote the restriction of $\tilde{\alpha}$ to $X$ by $\varphi$ :

$$
X \rightarrow \Xi \subset P, q_{1}+\cdots+q_{2 g-2}=D \mapsto \varphi(D)=\mathcal{O}_{\widetilde{C}}(D) .
$$

When $C$ is nonhyperelliptic, as we assume hereafter, $X$ is irreducible of dimension $p=\operatorname{dim}(P)=g-1=\tilde{g}-g$, and $\varphi$ maps $X$ onto the theta divisor $\Xi$ of $P$, with fibers isomorphic to odd dimensional projective spaces, with generic fiber dimension one. For further details and references on the Abel Prym map, consult S-V3].

Over a point $L$ of $\Xi$, the fiber of $\varphi$ is isomorphic to the linear series $|L|=$ $\mathbb{P} H^{0}(\widetilde{C}, L)$. Note that this fiber is smooth as a scheme, since the Abel Prym map is obtained by restricting the Abel map to the Prym divisor variety $X \subset \widetilde{C}^{(2 g-2)}$, and the fiber $|L|$ of $\tilde{\alpha}$ over $L$ is known to be smooth as a scheme.

Since $X$ is irreducible of dimension $p$, the normal cone $C_{|L|}(X)$ is of pure dimension $p$ (cf. [F] App. B.6.6]). Similarly, the tangent cone $C_{L}(\Xi)$ is of pure dimension $p-1$. In all cases, the normal cone $C_{|L|}(X)$ surjects onto the tangent cone $C_{L}(\Xi)$, and the goal of this paper is to examine some properties of this parametrization.

2.1.2. Exceptional points of $\Xi$. In the literature, there are a number of different classifications of singular points of the theta divisor $\Xi$ of a Prym variety. It is important to realize that the distinctions may depend on the choice of étale double cover $\pi: \widetilde{C} \rightarrow C$ defining the given principally polarized Prym variety $(P, \Xi)$. To fix notation, and highlight the various cases we study, we propose the following terminology. 
Definitions. Let $L \in \Xi \subset P \subset P i c^{2 g-2}(\widetilde{C})$. Suppose that the line bundle $L \cong$ $\pi^{*} M \otimes \mathcal{O}_{\widetilde{C}}(B)$ where $M \in \operatorname{Pic}(C)$ and $B$ is an effective divisor on $\widetilde{C}$. Assume that $h^{0}(M)$ is maximal, in the sense that whenever we write $L \cong \pi^{*} M^{\prime} \otimes \mathcal{O}_{\widetilde{C}}\left(B^{\prime}\right)$ where $M^{\prime} \in P i c(C)$ and $B^{\prime}$ is an effective divisor on $\widetilde{C}$, then $h^{0}(M) \geq h^{0}\left(M^{\prime}\right)$. We say the point $L$ is:

(1) exceptional if $h^{0}(M) \geq 2$,

(2) very exceptional if $h^{0}(M)>\frac{1}{2} h^{0}(L)$,

(3) totally exceptional if $h^{0}(M)=h^{0}(L)$.

Recall that if $L$ is not very exceptional, then mult $_{L} \Xi=\frac{1}{2} h^{0}(L)$ ([M, S-V5]), and if $L$ is very exceptional, then $\operatorname{mult}_{L} \Xi=h^{0}(M)$ ([CM] $)$.

Remarks. (i) The term "exceptional singularity" has been fairly standard for Mumford's case 1 in [M, p. 344]. For a connected étale double cover where $C$ is a Gieseker Petri general curve, there are no exceptional singularities of $\Xi$.

(ii) Recall that a singular point $L$ of $\Xi$ is called "stable" if $h^{0}(L) \geq 4$. The terms stable and exceptional as just defined are exhaustive for singular points of $\Xi$ but not mutually exclusive. The cases in common are those with $h^{0}(L) \geq 4$ and $h^{0}(M) \geq 2$. A singular point $L \in \Xi$ is not stable if and only if $h^{0}(L)=2$ and $L$ is totally exceptional.

2.1.3. Exceptional points of $X$. We can classify points $D \in X$ in a similar fashion. Suppose that the divisor $D=\pi^{*}(A)+B$ where $A$ is an effective divisor on $C$ and $B$ is an effective divisor on $\widetilde{C}$ such that $\operatorname{supp}(B) \cap \operatorname{supp}\left(\iota^{*} B\right)=\emptyset$. Here $\iota: \widetilde{C} \rightarrow \widetilde{C}$ is the involution associated with the étale double cover $\pi: \widetilde{C} \rightarrow C$, and the disjointness condition on the supports of $B$ and its "conjugate" $\iota^{*} B$ ensures that $A$ is maximal among effective divisors on $C$ with $\pi^{*}(A) \leq D$ on $\widetilde{C}$.

We say that $D$ is exceptional (resp. very exceptional, resp. totally exceptional) if $h^{0}(A) \geq 2\left(\right.$ resp. $h^{0}(A)>\frac{1}{2} h^{0}(D)$, resp. $\left.h^{0}(A)=h^{0}(D)\right)$.

Clearly a point $L \in \Xi$ is exceptional (resp. very exceptional, resp. totally exceptional) if and only if there exists $D \in|L| \subset X$ such that $D$ is exceptional (resp. very exceptional, resp. totally exceptional). Recall Beauville's formulation of Welters' criterion for smoothness of $X$ at $\widetilde{D}$ (cf. [S-V3, Lemma 2.13]): $X$ is singular at $D$ if and only if $D$ is exceptional.

2.1.4. The Mumford pairing. We will also want to use an equivalent characterization of exceptional points in terms of isotropic subspaces for the Mumford pairing. Consider $L \in \Xi$ and $s, t \in H^{0}(\widetilde{C}, L)$. Using the isomorphism $\iota^{*}(L) \cong$ $\Omega_{\widetilde{C}} \otimes L^{*}$, first let $\langle s, t\rangle$ in $H^{0}\left(\widetilde{C}, \Omega_{\widetilde{C}}\right)$ denote the cup product of $s$ and $\iota^{*}(t)$ by $H^{0}(L) \times H^{0}\left(\Omega_{\widetilde{C}} \otimes L^{*}\right) \rightarrow H^{0}\left(\Omega_{\widetilde{C}}\right)$. Then $H^{0}\left(\Omega_{\widetilde{C}}\right) \cong H^{0}\left(\Omega_{C}\right) \oplus H^{0}\left(\Omega_{C}(\eta)\right)$ and the skew-symmetric element $\langle s, t\rangle-\langle t, s\rangle$ lies in $H^{0}\left(\Omega_{C}(\eta)\right)$. The Mumford pairing is defined as

$$
\begin{gathered}
H^{0}(L) \times H^{0}(L) \stackrel{\beta}{\longrightarrow} H^{0}\left(\Omega_{C}(\eta)\right) \\
\beta(s, t)=\langle s, t\rangle-\langle t, s\rangle .
\end{gathered}
$$

We say that a subspace $\Lambda \subset H^{0}(L)$ is "isotropic" for this skew-symmetric pairing if $\beta(s, t)=0$ for all $s, t \in \Lambda$. Of course $\{0\}$ and all the 1-dimensional subspaces are isotropic. If $L$ can be expressed as $\pi^{*} M \otimes \mathcal{O}_{\widetilde{C}}(B)$, for $M \in \operatorname{Pic}(C)$ with $h^{0}(M) \geq 2$, and $B$ an effective divisor on $\widetilde{C}$ defined by $u \in H^{0}\left(\mathcal{O}_{\widetilde{C}}(B)\right)$, then it is easy to see that $\Lambda=\pi^{*} H^{0}(M) \cdot u$ is an isotropic subspace of $H^{0}(L)$ of dimension $h^{0}(M)$. It is 
shown in $[\mathrm{S}-\mathrm{V} 5]$ that when $\operatorname{supp}(B) \cap \operatorname{supp}\left(\iota^{*} B\right)=\emptyset$, then this subspace is maximal isotropic in the sense that it is not properly contained in another isotropic subspace; and conversely, any maximal isotropic subspace of $H^{0}(L)$ of dimension $\geq 2$ has this form. Thus $L \in \Xi$ is exceptional (resp. very exceptional, resp. totally exceptional) if and only if $\beta$ admits an isotropic subspace of dimension $\geq 2$ (resp. $>\frac{1}{2} h^{0}(L)$, resp. $\left.=h^{0}(L)\right)$.

It follows that the Mumford pairing has the following "Isotropy Property": if $V_{1}, V_{2} \subset H^{0}(L)$ are isotropic subspaces and $V_{1} \cap V_{2} \neq 0$, then $V_{1}+V_{2}$ is isotropic [S-V5, Lemma 2.3 (iii)]. In particular, every nonzero isotropic subspace is contained in a unique, maximal isotropic subspace.

Thus the pairing $\beta$ induces an equivalence relation on the divisors in the linear system $|L|=\mathbb{P} H^{0}(L)$. The graph of the equivalence relation is the locus of all pairs $([v],[w]) \in \mathbb{P} H^{0}(L) \times \mathbb{P} H^{0}(L)$ with $\beta(v, w)=0$, hence is algebraic, and each equivalence class is a linear subspace of $\mathbb{P} H^{0}(L)$. Now there appears a basic dichotomy: if the pairing $\beta$ is identically 0 (equivalently $L \in \Xi$ is "totally exceptional"), then there is only one equivalence class, namely $\mathbb{P} H^{0}(L)$, occurring as the unique maximal isotropic linear subspace, and every point of $|L|$ is singular on $X$. Otherwise, we claim that even the union of all the maximal isotropic linear subspaces of positive dimension is a proper algebraic subset of $|L|$; that is, equivalently, the general point of $|L|$ is nonsingular on $X$.

Proposition. For an algebraic equivalence relation on $\mathbb{P}^{r}$, if all equivalence classes are proper linear subspaces, then the general equivalence class is a point.

Proof. The statement holds for $\mathbb{P}^{2}$ since any two lines in a projective plane could not be disjoint equivalence classes. If the statement fails for $r>2$ and the general equivalence class is of positive dimension, then restrict the equivalence relation to a general hyperplane $H$ in $\mathbb{P}^{r}$. We get an algebraic equivalence relation on $H \cong \mathbb{P}^{r-1}$ with all equivalence classes proper linear subspaces, and the general equivalence class has dimension one less than before. By induction on $r$ we may assume that the general equivalence class in $H$ is a point. Then choose a hypersurface $C$ in $H$ containing a general point and then a general point $p$ in $H-C$. Look at the union of the equivalence classes in $\mathbb{P}^{r}$ which intersect $H$ along the hypersurface $C$. This union forms a hypersurface $K$ in $\mathbb{P}^{r}$ and is disjoint from the equivalence class of $p$ in $\mathbb{P}^{r}$, which is a line meeting $H$ at $p$. But a hypersurface in $\mathbb{P}^{r}$ cannot be disjoint from a line, a contradiction.

2.2. The parametrization induced by $\varphi$ for a tangent cone to $\Xi$. Let $L$ be any point on $\Xi$, and $|L|$ the fiber of the Abel Prym map $\varphi$ over it. Then the projective normal cone to $|L|$ in $X$ maps onto the projective tangent cone at $L$ to $\Xi$ by the map induced by blowing up the subvarieties $|L|$ and $\{L\}$ in $X$ and $\Xi$ respectively:

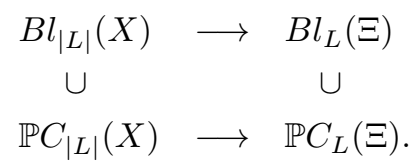

Moreover, $X$ is contained in the smooth variety $\widetilde{C}^{(2 g-2)}$, and the map on the blowup of $|L|$ in $X$ is the restriction of the map induced by blowing up $|L|$ in $\widetilde{C}^{(2 g-2)}$ and 
$\{L\}$ in $\widetilde{\Theta}$ :

$$
\begin{array}{ccc}
B l_{|L|}\left(\widetilde{C}^{(2 g-2)}\right) & \longrightarrow & B l_{L}(\widetilde{\Theta}) \\
\cup & \cup \\
B l_{|L|}(X) & \longrightarrow & B l_{L}(\Xi) .
\end{array}
$$

Since both $|L|$ and $\widetilde{C}^{(2 g-2)}$ are smooth, the map on $B l_{|L|}\left(\widetilde{C}^{(2 g-2)}\right)$ is given by the differential of the Abel map on $\widetilde{C}^{(2 g-2)}$. Thus every point of the normal cone to $|L|$ in $X$ is represented by a Zariski tangent vector to $\widetilde{C}^{(2 g-2)}$ at a point $D \in|L|$, and the map from the normal cone of $|L|$ in $X$ to the tangent cone of $\Xi$ at $L$ is induced by the Abel differential acting on the Zariski tangent space $T_{D}\left(\widetilde{C}^{(2 g-2)}\right)$.

2.2.1. The differential of the Abel Prym map. For background on the differential $\tilde{\alpha}_{*}: T_{D}\left(\widetilde{C}^{(2 g-2)}\right) \rightarrow T_{L}(\widetilde{J})$ of the Abel map $\tilde{\alpha}: \widetilde{C}^{(2 g-2)} \rightarrow \widetilde{J}$ at a point $D \in \widetilde{C}^{(2 g-2)}$, see $\mathrm{M}-\mathrm{M}, \mathrm{K} 1, \mathrm{~K} 3, \mathrm{ACGH}$. According to the summary in ACGH, p. 191, with $d=2 g-2$ and $r=0$ ], the image of the Abel differential at $D$ is the subspace $W=$ $\tilde{\alpha}_{*}\left(T_{D}\left(\widetilde{C}^{(2 g-2)}\right)\right)$ of $T_{L}(\widetilde{J}) \cong H^{1}\left(\widetilde{C}, \mathcal{O}_{\widetilde{C}}\right)$ with "orthogonal complement" $W^{\perp}=$ $H^{0}\left(\Omega_{\widetilde{C}}(-D)\right)$ of $H^{0}\left(\widetilde{C}, \Omega_{\widetilde{C}}\right) \cong T_{L}^{*}(\widetilde{J})$. That is, the regular differential 1-forms on $\widetilde{C}$ that vanish on the divisor $D$ represent precisely the linear forms that vanish on the Abel image subspace; if the section $s$ of $L=\mathcal{O}_{\widetilde{C}}(D)$ defines $D$, then this linear form subspace $W^{\perp}$ is the image of cup product with $s$ :

$$
H^{0}\left(\Omega_{\widetilde{C}} \otimes L^{*}\right) \stackrel{s \cup}{\longrightarrow} H^{0}\left(\widetilde{C}, \Omega_{\widetilde{C}}\right) .
$$

The image of the differential of the Abel Prym map is simply the image of the restriction of the differential of the Abel map to the tangent space to $X$, since the divisor variety $X$ is the inverse image of $P$ under the Abel map. Now the tangent and cotangent spaces of our Prym variety $P$ at any point $L$ are isomorphic to those of the abelian variety $P_{0}$ at the origin 0 , under canonical isomorphisms of the tangent and cotangent spaces of $\widetilde{J}$ at $L$ to those of the abelian variety $\widetilde{J}_{0}$ at 0 . The Prym tangent and cotangent spaces, $T_{0}\left(P_{0}\right) \cong H^{1}\left(C, \mathcal{O}_{C}(\eta)\right)$ and $T_{0}^{*}\left(P_{0}\right) \cong$ $H^{1}\left(C, \Omega_{C}(\eta)\right)$ respectively, consist of exactly the skew-symmetric elements in the Jacobian tangent and cotangent spaces $T_{0}\left(\widetilde{J}_{0}\right)$ and $T_{0}^{*}\left(\widetilde{J}_{0}\right)$ respectively, for the induced action of the involution $\iota$ of $\widetilde{C}$.

Therefore, for $D \in X, L=\mathcal{O}_{\widetilde{C}}(D)$, and $s \in H^{0}(L)$ defining $D$, the analogous description of the image in $T_{L}(P)$ of the Abel Prym differential by linear forms on $T_{L}(P)$ will result by restricting the linear forms from $T_{L}(\widetilde{J})$ to $T_{L}(P)$, and the restriction map is implemented by skew-symmetrization. Thus, for $t \in H^{0}(L)$, and hence $\iota^{*}(t) \in H^{0}\left(\Omega_{\widetilde{C}} \otimes L^{*}\right)$, the linear form $s \cup \iota^{*}(t)$ (which we abbreviate as $\langle s, t\rangle$ ) on $T_{L}(\widetilde{J})$ restricts to $\frac{1}{2}$ the linear form $s \cup \iota^{*}(t)-t \cup \iota^{*}(s)=\langle s, t\rangle-\langle t, s\rangle=\beta(s, t)$. Finally, upon replacing $H^{0}\left(\Omega_{\widetilde{C}} \otimes L^{*}\right)$ by $H^{0}(L)$ for $L=\varphi(D) \in \Xi$, and passing from $H^{0}\left(\widetilde{C}, \Omega_{\widetilde{C}}\right)$ to $H^{0}\left(\Omega_{C}(\eta)\right)$ by skew-symmetrizing, exactly as in Mumford's paper [M] p. 343], we reach the following conclusion, which we state for reference.

Proposition. For a connected étale double cover $\pi: \tilde{C} \rightarrow C$ of a smooth curve $C$ of genus $g$, with Prym variety $(P, \Xi)$ and Abel Prym map $\varphi: X \rightarrow \Xi \subset P$, consider any point $D \in X$ and its image point $L=\varphi(D) \in \Xi$. Let $s$ be a section of the line bundle $L=\mathcal{O}_{\widetilde{C}}(D)$ defining the divisor $D$ on $\widetilde{C}$. Then the image $\varphi_{*}\left(T_{D}(X)\right)$ of the differential of the Abel Prym map at $D \in X$ is the subspace of $T_{L}(P)$ given as the 
orthogonal complement of the image of

$$
\beta(s,-): H^{0}(L) \rightarrow H^{0}\left(\Omega_{C}(\eta)\right)
$$

under the duality between $T_{L}(P) \cong H^{1}\left(C, \mathcal{O}_{C}(\eta)\right)$ and $T_{L}^{*}(P) \cong H^{0}\left(\Omega_{C}(\eta)\right)$.

Since the Abel Prym differential sends the subspace $T_{D}(|L|)$ to 0 in $T_{L}(P)$, it induces a linear map on the normal vector space $\left.N_{|L|}(X)\right|_{D} \cong T_{D}(X) / T_{D}(|L|)$. In fact, $T_{D}(|L|)$ is exactly the kernel of the Abel Prym differential $T_{D}(X) \rightarrow T_{L}(P)$, so we get an injection on the normal vector space $\left.N_{|L|}(X)\right|_{D} \hookrightarrow T_{L}(P)$. The tangent space $T_{D}(X)$ has dimension $p+\operatorname{dim}(\Lambda)-1$ where $\Lambda$ is the maximal isotropic subspace of $H^{0}(L)$ containing a section $s$ defining $D$ [S-V3, Corollary 2.14].

2.2.2. Normal cone and normal space. Globally there is a total normal space $N_{|L|}(X)$ of $X$ along $|L|$ and a morphism $\varphi_{*}: N_{|L|}(X) \rightarrow T_{L}(P)$ inducing the previous injective linear map on the fiber over each $D \in|L|$. This total space is a closed subscheme of $|L| \times T_{L}(P)$. Thus, from the Mumford pairing $\beta$, we have a rather satisfactory description of the map $N_{|L|}(X) \rightarrow T_{L}(P)$.

However, the complication is that it is the normal cone $C_{|L|}(X)$ and the maps of its components onto the tangent cone $C_{L}(\Xi)$ that we wish to determine. The fiber $\left.C_{|L|}(X)\right|_{D}$ of the normal cone $C_{|L|}(X)$ at a point $D \in X$ is a closed subscheme of the fiber $\left.N_{|L|}(X)\right|_{D}$ of the normal space at $D$. The fiber of the normal space is quite predictable: $\left.N_{|L|}(X)\right|_{D} \cong T_{D}(X) / T_{D}(|L|)$, while it may be difficult to determine $\left.C_{|L|}(X)\right|_{D}$ even set-theoretically. Globally the normal cone $C_{|L|}(X)$ is a closed subscheme of the normal space $N_{|L|}(X)$, and this inclusion of schemes over $|L|$ may be described briefly as follows. For any affine open subset $\mathcal{U} \subset X$, let $A$ be the coordinate ring of $\mathcal{U}$ and let $I \subset A$ be the ideal of $U=|L| \cap \mathcal{U}$. Then, over $U$, the inclusion $\left.\left.C_{|L|}(X)\right|_{U} \subset N_{|L|}(X)\right|_{U}$ of affine schemes is defined by the surjection $g r_{I}(A) \nleftarrow \operatorname{sym}\left(I / I^{2}\right)$ of graded $A / I$-algebras, from the symmetric algebra on $I / I^{2}$ to the associated graded of $A$ with respect to $I$, i.e. the natural homomorphism: $A / I \oplus I / I^{2} \oplus I^{2} / I^{3} \oplus \cdots \leftarrow A / I \oplus I / I^{2} \oplus \operatorname{sym}^{2}\left(I / I^{2}\right) \oplus \ldots$

2.2.3. The previously known (nonexceptional) case. If $L \in \Xi$ is not an exceptional singularity, i.e. $H^{0}(L)$ has no isotropic subspaces of dimension 2 or more, then the divisor variety $X$ is smooth along $|L|$. Then $C_{|L|}(X)=N_{|L|}(X)$ is a vector bundle of rank $p-\left(h^{0}(L)-1\right)$ over $|L|$ (and hence is smooth, irreducible), and the normal bundle $N_{|L|}(X)$ maps onto the tangent cone $C_{L}(\Xi)$. This is proved in our paper S-V3, Cor. 2.8]. Thus, in the nonexceptional case, the projectivized tangent cone $\mathbb{P} C_{L}(\Xi) \subset \mathbb{P}^{p-1}$ is an irreducible hypersurface of degree $\frac{1}{2} h^{0}(L)$, swept out by a family of linear spaces (all of the same dimension) parametrized by $|L|$.

In particular, if $L \in \Xi$ is a smooth point, $C_{L}(\Xi)=T_{L}(\Xi)$ is a hyperplane $H \subset T_{L}(P)$, and the normal bundle $N_{|L|}(X) \subset|L| \times T_{L}(P)$ is equal to $|L| \times H$, and hence is trivial over $|L| \cong \mathbb{P}^{1}$.

\section{The EXPECTED TYPICAL STRUCTURE FOR EXCEPtIONAL SINGULARITIES}

3.1. The three different types of exceptional singularities. The different types of exceptional singular points $L$ on a Prym theta divisor $\Xi$ can be listed as follows:

(1) exceptional but not very exceptional singular point,

(2) very exceptional but not totally exceptional singular point,

(3) totally exceptional singular point. 
For each of the three cases we review the properties that are known to hold and formulate the simplest structure that the parametrization $C_{|L|}(X) \rightarrow C_{L}(\Xi)$ could possibly have. Thus, consider $L \in \Xi$, and set $n=h^{0}(L)$ so that $|L| \cong \mathbb{P}^{n-1}$.

(1) If $L$ is an exceptional but not very exceptional singular point of $\Xi$, then $n \geq 4$, and the divisor variety $X$ has some singularities in $|L|$ while the general point of $|L|$ is smooth on $X$. Thus $N_{|L|}(X)$ fails to be a vector bundle over $|L|$, but the fiber over a generic point does have dimension $p-(n-1)$. The normal space $N_{|L|}(X)$ maps onto the tangent cone $C_{L}(\Xi)$ and Mumford's Pfaffian equation of degree $n / 2$ defines $C_{L}(\Xi) \subset T_{L}(P)$ (so we say that "RST" holds at $L$ ). Surprisingly however, the unique component of $N_{|L|}(X)$ and $C_{|L|}(X)$ that dominates $|L|$ may fail to surject onto $C_{L}(\Xi)$; see section 7 for an example.

(2) If $L$ is a very exceptional but not totally exceptional singular point of $\Xi$, then $n \geq 4$, the divisor variety $X$ is singular (at least) along a distinguished projective linear subspace $S \subset|L|$ of dimension $m-1$ with $n / 2<m<n$, and $X$ is smooth at a general point of $|L|$. Thus, since $n$ is even, then $m-1>\frac{1}{2}(n-1)$ and this maximal isotropic linear subspace $S$ of singular points of $X$ in the fiber $|L|$ of the Abel Prym map has more than half the dimension of the projective space $|L|$. Mumford's Pfaffian polynomial is identically 0 . The normal cone $C_{|L|}(X)$ is reducible; see Theorem 2 in section 5. The unique irreducible component dominating $|L|$ does not map onto an irreducible component of $C_{L}(\Xi)$, so there is at least one irreducible component supported over $S$ (dominating either all of $S$ or a subvariety) that maps onto an irreducible component of $C_{L}(\Xi)$. Furthermore, the restriction of $N_{|L|}(X)$ to $S$ is a vector bundle of rank $p+m-n$ over $S$ and any irreducible component of $C_{|L|}(X)$ supported over $S$ is properly contained in this vector bundle.

(3) If $L$ is a totally exceptional singular point of $\Xi$, then $X$ is singular everywhere along $|L|$. Mumford's skew symmetric matrix of linear forms is identically 0 . Hence $N_{|L|}(X)$ is the trivial vector bundle $|L| \times T_{L}(P)$ of rank $p$ over $|L|$; see section 4 .

3.2. The simplest possible structure for the parametrization. What is the expected typical structure in cases $(1),(2),(3)$, unless some degeneracy occurs?

In (1) the simplest possibility is for $N_{|L|}(X)$ to be irreducible. Then it would follow that $C_{|L|}(X)=N_{|L|}(X)$ and sweeps out $C_{L}(\Xi)$ by a family of linear spaces (not all of the same dimension) parametrized by $|L|$. Indeed, whenever $L$ is not totally exceptional then the normal space $N_{|L|}(X)$ has an irreducible component of dimension $p$ supported over all of $|L|$, according to the Proposition in 2.1.4 and the known properties of $N_{|L|}(X)$ reviewed in 4.1 below. Since the normal cone $C_{|L|}(X)$ also has an irreducible component of dimension $p$ supported over all of $|L|$, the normal cone and normal space have this standard component in common. See the examples of stable double points of rank 4,5 , or 6 in section 4.2 .

In (2) the simplest possibility is for $C_{L}(X)$ to have 2 irreducible components, one dominating $|L|$ and the other dominating $S$, with the latter component sweeping out $C_{L}(\Xi)$ by a family of $p-(m-1)$ dimensional subvarieties parametrized by $S$. See Theorem 3 in section 6.1 .

In (3) the simplest possibility is for $C_{|L|}(X)$ to be irreducible and sweep out $C_{L}(\Xi)$ by a family of $p-(n-1)$ dimensional varieties parametrized by $|L|$. See the examples of totally exceptional pencils in sections 6.3.1, 6.3.2, and 6.4.

Questions. (i) For an exceptional singular point $L$ on a Prym theta divisor, which subvarieties of $|L|$ are dominated by the irreducible components of the normal cone 
$C_{|L|}(X)$ of the divisor variety $X$ ? That is, identify the "distinguished subvarieties" (in the sense of [F, Def. 6.1.2, pp. 94-95]) of the linear series $|L| \subset X$.

(ii) For the Prym variety $(P, \Xi)$ of a connected étale double cover of a nonhyperelliptic smooth curve $C$, are all the tangent cones of $\Xi$ irreducible?

Remark. In Prym canonical space $\mathbb{P} T_{0}\left(P_{0}\right) \cong \mathbb{P}^{p-1}$ consider both the Prym canonical model of the curve $C$ (assumed nonhyperelliptic) and the hypersurface gotten by translating the projectivized tangent cone $\mathbb{P} C_{L}(\Xi)$ from $L$ to the origin in the abelian variety $P_{0}$. Casalaina-Martin [CM, Cor. 6.2.4, p. 200] has shown that the Prym canonical curve $C$ is always contained in $\mathbb{P} C_{L}(\Xi)$ when $h^{0}(L) \geq 4$, and never when $h^{0}(L)=2$ and $L$ is (totally) exceptional.

Notation for the numerical type of a very exceptional singularity. For any very exceptional singular point $L \in \Xi$, with the standard unique expression $L=\pi^{*}(M)(B)$, we use the pair $(m, n)$ to indicate that $m=h^{0}(M)$ and $n=h^{0}(L)$. Thus $n \geq 2$ is even, $\frac{n}{2}<m \leq n$, and $m$ is the largest dimension of an isotropic subspace of $H^{0}(L)$ for the pairing $\beta: H^{0}(L) \times H^{0}(L) \rightarrow T_{0}^{*}\left(P_{0}\right)$.

Question. What is the basic geometry associated with each numerical type $(m, n)$ of a very exceptional singularity, and what degeneracies can occur?

Remark on another invariant. In describing the geometry, it may be helpful to take into account also $m^{\prime}=h^{0}(M(\eta))$, with $0 \leq m^{\prime} \leq n-m$. For instance, it seems that when $m=n-1$ and $m^{\prime}=1$, the basic model generalizing the main example of this paper should be that the projectivized tangent cone $\mathbb{P} C_{L}(\Xi) \subset \mathbb{P}^{p-1}$ will be swept out by an $(n-2)$-dimensional family of hyperplane sections parametrized by $|M|$ and consisting of all the hyperplanes of Prym canonical space containing a codimension $n-1$ linear space.

Remark on $(n-1, n)$. In this case $X$ must be smooth at every point of $|L|-$ $\left(\pi^{*}|M|+B\right)$. (Indeed, following [S-V6], if $D \in|L|$ is a singular point of $X$, then there is at least an isotropic $\mathbb{P}^{1}$ through $D$. But a line in $|L| \cong \mathbb{P}^{n-1}$ must meet $\pi^{*}|M|+B \cong \mathbb{P}^{n-2}$, and hence in fact be contained in $\pi^{*}|M|+B$.) Consider the irreducible component $\mathcal{C}_{1}$ of the normal cone $C_{|L|}(X)$ that dominates $|L|$ and the map from $\mathcal{C}_{1}$ to $T_{L}(P)$. By the arguments in the proof of Theorem 2 in section 5 , for each smooth point $D$ of $X$ in $|L|$, the linear map from the fiber over $D$ in $\mathcal{C}_{1}$ to $T_{L}(P)$ is an isomorphism onto a codimension $n-1$ subspace $V \subset T_{L}(P)$, and in this case the image subspace $V$ cannot vary with $D$. It follows that $\mathcal{C}_{1}=|L| \times V \subset$ $|L| \times T_{L}(P)$, generalizing the observed behavior for the cubic threefold case (for which $\mathbb{P}(V)$ is the line $\ell$ on the cubic threefold in $\left.\mathbb{P}^{4}\right)$.

Remarks on $(3,4)$. It is natural to ask for some other cases in which $(3,4)$ occurs, besides that of intermediate Jacobians of cubic threefolds. Following B2, Rem. 6.27, pp. 377-378], let $W \subset \mathbb{P}^{d-1}$ be an odd dimensional cubic hypersurface containing a linear subspace $\mathbb{P}^{d-4}$, with $d \geq 5$. For $d=5$ this is the cubic threefold case, with a marked line. But for $d \geq 7$ such a cubic hypersurface is singular. So assume that the blowup $\widetilde{W}$ of $W$ along the $\mathbb{P}^{d-4}$ subvariety is smooth, and moreover that the degree $d$ discriminant curve $C \subset \mathbb{P}^{2}$ is smooth. Then [B2] there is an étale double cover $\pi: \widetilde{C} \rightarrow C$ for which the Prym variety is isomorphic to the intermediate Jacobian of $\widetilde{W}$.

We carried out a preliminary investigation for cubic fivefolds $\mathbb{P}^{3} \subset W \subset \mathbb{P}^{6}$, i.e. $d=7$. The theta divisor then has an irreducible 5-dimensional family of very 
exceptional triple points, expressible as $\pi^{*}\left(g_{7}^{2}\right)+B$ where $B$ lies over a divisor in the linear series $\left|\mathcal{O}_{C}(2)\right|$ cut by conics on the plane septic curve $C$. It seems reasonable to hope to obtain a Torelli result by using the tangent cone at a general such triple point.

Remarks on $(n, n)$. (i) For any totally exceptional singularity with $n=2$, i.e. a "totally exceptional pencil" $|L| \cong \mathbb{P}^{1}$, then for some Zariski open neighborhood $U$ of $L$ in $\Xi$, the preimage $\varphi^{-1}(U)$ in $X$ is isomorphic to $\mathbb{P}^{1} \times U$ so the normal cone $C_{|L|}(X)$ is a product $\mathbb{P}^{1} \times C_{L}(\Xi)$. Indeed, the map $\varphi^{-1}(U) \rightarrow U$ is pulled back from a local model which has this Zariski local triviality property around points with a $\mathbb{P}^{1}$ fiber; cf. [S-V6, section 5] and the map $\mathbb{K} \rightarrow \Sigma$ described in the next section here.

(ii) We do not know the structure of the tangent cone at a general totally exceptional singularity $L$, except that from $[\mathrm{CM}$ the multiplicity is $n$ and hence from S-V6] the tangent cone is defined by the Pfaffian of an $n \times n$ skew-symmetric matrix of quadratic forms on $T_{L}(P)$.

\section{The GeOMEtry of Mumford's SKEW-Symmetric MATRIX OF LINEAR FORMS}

4.1. The normal space as a pullback. For $\varphi: X \rightarrow \Xi$ and $L \in \Xi$ we are interested in the parametrization $C_{|L|}(X) \rightarrow C_{L}(\Xi)$ of the tangent cone by the normal cone along the fiber. But how much of the structure is already determined at 1 st order, i.e. by the Abel Prym map derivative $\varphi_{*}: N_{|L|}(X) \rightarrow T_{L}(P)$ on the normal space along the fiber? Thus, consider the diagram:

$$
\begin{array}{ccrl}
|L| \subset X & & |L| \leftarrow N_{|L|}(X) & \supseteq C_{|L|}(X) \\
\mid & \downarrow \varphi & \varphi_{*} \downarrow & \downarrow \\
L \in \Xi \subset P & & T_{L}(P) & \supset C_{L}(\Xi)
\end{array}
$$

According to Kempf and Mumford, $N_{|L|}(X) \subset|L| \times T_{L}(P)$ is pulled back from a universal linear algebra model, where $N_{|L|}(X)$ is embedded in $|L| \times T_{L}(P)$ by the product of the projection map $N_{|L|}(X) \rightarrow|L|$ and $\varphi_{*}: N_{|L|}(X) \rightarrow T_{L}(P)$. We will review a formulation of this result. Throughout we may use the canonical identification of the tangent spaces of $P$ with the tangent space $T_{0}\left(P_{0}\right)$ by translation to the origin, and we will drop the subscript on $P_{0}$. Thus we will write $T_{L}(P)$ or $T_{0}(P)$, whichever one seems better in each instance.

The skew-symmetric bilinear form $\beta: H^{0}(L) \times H^{0}(L) \rightarrow H^{0}\left(\Omega_{C}(\eta)\right)$ can be represented with respect to a basis for $H^{0}(L)$ as a skew-symmetric matrix $M_{1}$ of linear forms on $T_{0}(P)$, since $H^{0}\left(\Omega_{C}(\eta)\right) \cong T_{0}^{*}(P)$. Thus, with $n=h^{0}(L)$, let $\operatorname{Alt}(n)$ be the vector space of all $n \times n$ skew-symmetric matrices over $\mathbb{C}$. Then $M_{1}$ defines a linear map

$$
T_{L}(P) \rightarrow \operatorname{Alt}(n),
$$

so that if we identify $|L|=\mathbb{P}^{n-1}$ (from $H^{0}(L) \cong \mathbb{C}^{n}=$ column vectors, w.r.t. to our basis), then we get a map on the product

$$
|L| \times T_{L}(P) \stackrel{\left(\text { ident., } M_{1}\right)}{\longrightarrow} \mathbb{P}^{n-1} \times \operatorname{Alt}(n) .
$$

Now define

$$
\mathbb{K}=\left\{([\sigma], A) \in \mathbb{P}^{n-1} \times \operatorname{Alt}(n) \mid A \sigma=0\right\},
$$

where $[\sigma]$ denotes the point of $\mathbb{P}^{n-1}$ determined by a nonzero column vector $\sigma \in \mathbb{C}^{n}$. 
Theorem (Kempf and Mumford). $N_{|L|}(X)=\left(\text { ident., } M_{1}\right)^{-1}(\mathbb{K})$ as subschemes of $|L| \times T_{L}(P)$.

Proof. We can reference Kempf [K1, Lemma, p. 183], in the language of "wellpresented families of projective spaces", for the corresponding 1st order statement and proof in the Jacobian case. That is, since $L$ is a point of the theta divisor of the Jacobian $\widetilde{J}$ of the curve $\widetilde{C}$, one has the geometry associated with the RiemannKempf matrix, which is an $n \times n$ matrix of linear forms on $T_{0}(\widetilde{J})$ and is never skew-symmetric. Then Mumford [M, pp. 342-343] showed how to restrict all the 1st order objects to the Prym case.

Corollary 1 (equivalent to the Proposition in 2.2.1). For $L \in \Xi$, let $s$ be a nonzero section of the line bundle $L$ on $\widetilde{C}$ and let $[s] \in|L| \subset X$ denote the divisor of $s$. In terms of a basis for $H^{0}(L)$, if $s$ is expressed as a column vector and $M_{1}$ as a skew-symmetric matrix of linear forms on $T_{0}(P)$, then the image in $T_{L}(P) \cong T_{0}(P)$ of the fiber of $N_{|L|}(X)$ at the point $[s]$, i.e. of the Zariski normal space to $|L|$ in $X$ at $[s]$, is the common base locus in $T_{0}(P)$ of the column vector of linear forms $M_{1} s$. Intrinsically, the equivalent statement is that the image $\varphi_{*}\left(\left.N_{|L|}(X)\right|_{[s]}\right) \subset T_{L}(P) \cong$ $T_{0}(P)$ is equal to $\left\{v \in T_{0}(P) \mid \beta(s,-)(v)=0\right.$ (as an element of $\left.\left.H^{0}(L)^{*}\right)\right\}$.

Proof. A vector $v$ in $T_{0}(P)$ is in that common base locus if and only if the column vector $\left(M_{1} s\right)(v)=M_{1}(v) s=0$, iff $s$ is in the kernel of $M_{1}(v)$, iff $\left([s], M_{1}(v)\right)$ is in $\mathbb{K}$, if and only if $([s], v)$ is in $N_{|L|}(X)$.

Corollary 2. Every irreducible component of the normal space $N_{|L|}(X)$ has dimension at least $p=\operatorname{dim}(P)$.

Proof. By the Theorem, $N_{|L|}(X)$ is the inverse image of a smooth subvariety of codimension $n-1$ in a smooth variety. Hence every irreducible component of $N_{|L|}(X)$ has codimension at most $n-1=\operatorname{dim}|L|$ in $|L| \times T_{L}(P)$, i.e. dimension $\geq p$.

Remark. In principle the results summarized here have been completely known at least since 1974. The higher order statement in the Jacobian case is given with references in [K1, Thm., p. 183] and a proof is given in [K3, Thm. 21.4, p. 189]; that is, a Jacobian theta divisor has a local determinantal equation which gives rise to the equation for the tangent cone as a determinant of linear forms. The clearest possible treatment may be the exposition by Kempf [K2, pp. 1-9 of the 2nd section] describing the original approach of Mumford and Kempf to Riemann's singularity theorem. One may consult our paper [S-V6, sections 3-4] for some review and formulations. The main point of [S-V6] was to refine the known existence theory for a local inducing map from the Jacobian $\widetilde{J}$ to matrix space to get the existence S-V6, Thm., p. 225] of a local inducing map from the Prym $P$ to skew-symmetric matrix space. It is the 1 st order part, i.e. the derivative at $L$ of a nonlinear map, that always has an intrinsic description as the linear map

$$
T_{L}(P) \longrightarrow \Lambda^{2}\left(H^{0}(L)^{*}\right) \cong\left\{\text { alternating bilinear forms on } H^{0}(L)\right\}
$$

dual to Mumford's linear map ( $\underline{\mathrm{M}}$, display just below the middle of p. 343])

$$
T_{0}^{*}(P) \cong H^{0}\left(\Omega_{C}(\eta)\right) \longleftarrow \Lambda^{2} H^{0}(L) .
$$

Thus, in terms of a basis for $H^{0}(L)$, if we identify the alternating bilinear forms on $H^{0}(L)$ with the skew-symmetric $n \times n$ matrices $\operatorname{Alt}(n)$ (and also equate $T_{0}(\operatorname{Alt}(n)$ ) 
with $\operatorname{Alt}(n))$, then we say simply that Mumford's skew-symmetric matrix of linear forms on $T_{0}(P)$ expresses this derivative at $L$ as $T_{L}(P) \rightarrow \operatorname{Alt}(n), z \mapsto M_{1}(z)$ by evaluating all the entries at $z \in T_{L}(P) \cong T_{0}(P)$.

Now we look at the corresponding linear algebra diagram:

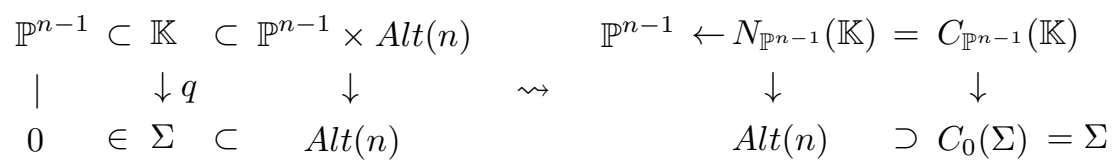

Here $\Sigma$ is the locus in $\operatorname{Alt}(n)$ consisting of all singular $n \times n$ skew-symmetric matrices. Precisely, $\Sigma \subset \operatorname{Alt}(n)$ is the closed subscheme defined by the equation $\operatorname{Pf}(A)=0$, where the Pfaffian $P f$ is a homogeneous polynomial function of degree $n / 2$ on $\operatorname{Alt}(n)$ whose square is the determinant. $\Sigma$ is a conical hypersurface (with vertex at 0 ) since $\operatorname{Pf}(A)$ is homogeneous in $A$, and is reduced and irreducible.

The variety $\mathbb{K}$ is smooth, and its closed subscheme $\{([\sigma], 0)\} \cong \mathbb{P}^{n-1}$ is smooth, so the normal space $N_{\mathbb{P}^{n-1}}(\mathbb{K})$ is a vector bundle over $\mathbb{P}^{n-1}$ and is equal to the normal cone $C_{\mathbb{P}^{n-1}}(\mathbb{K})$ of $\mathbb{K}$ along $\mathbb{P}^{n-1}$. The tangent cone of $\Sigma$ at 0 is $\Sigma$ itself: $C_{0}(\Sigma)=\Sigma\left(\right.$ under the canonical vector space identification $\left.T_{0}(\operatorname{Alt}(n))=\operatorname{Alt}(n)\right)$. Similarly, the normal bundle $N_{\mathbb{P}^{n-1}}(\mathbb{K}) \subset \mathbb{P}^{n-1} \times \operatorname{Alt}(n)$ is already exactly $\mathbb{K}$ itself.

Thus the parametrization $q: \mathbb{K} \rightarrow \Sigma,([\sigma], A) \mapsto A$ (induced by the projection $\mathbb{P}^{n-1} \times \operatorname{Alt}(n) \rightarrow \operatorname{Alt}(n)$ to the 2 nd factor), can also be interpreted as the parametrization $N_{\mathbb{P}^{n-1}}(\mathbb{K}) \rightarrow C_{0}(\Sigma)$ of the tangent cone by the normal cone (or space, or bundle) along the fiber.

In summary, the map $N_{|L|}(X) \rightarrow T_{L}(P)$ is equivalent to the pullback of the map $q$ (viewed as $\mathbb{K}=N_{\mathbb{P}^{n-1}}(\mathbb{K}) \rightarrow C_{0}(\Sigma)=\Sigma \subset$ Alt $(n)$ ) by the linear map $T_{L}(P) \rightarrow \operatorname{Alt}(n):$

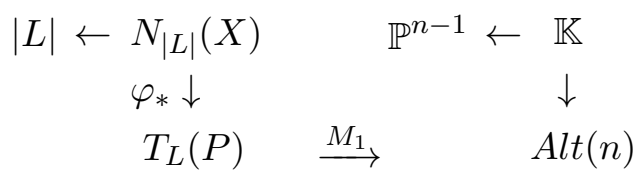

In other words, the Abel Prym derivative on the normal space of $X$ along $|L|$ is induced - by Mumford's skew-symmetric matrix of linear forms - from the completely standard (projective) "kernel fibration" $q: \mathbb{K} \rightarrow \Sigma \subset \operatorname{Alt}(n)$. For any $A \in \operatorname{Alt}(n)$ the fiber of $q$ is the projectivized null space $\mathbb{P} k e r(A)$, and the generic fiber over $\Sigma$ is a $\mathbb{P}^{1}$.

Now we signal exactly what is parametrized by the normal space $N_{|L|}(X)$. The result [S-V3, Cor. 2.8, p. 489] is that $N_{|L|}(X)$ always parametrizes the restriction of the Jacobian tangent cone $C_{L}(\widetilde{\Theta})$ to the Prym tangent space $T_{L}(P)$; that is,

$$
\varphi_{*}\left(N_{|L|}(X)\right)=C_{L}(\widetilde{\Theta}) \cap T_{L}(P) .
$$

It follows that either $C_{L}(\widetilde{\Theta}) \cap T_{L}(P)=C_{L}(\Xi)$ as sets or else $C_{L}(\widetilde{\Theta}) \supset T_{L}(P)$. In the first case (that is, when $C_{L}(\widetilde{\Theta}) \cap T_{L}(P)=C_{L}(\Xi)$ as sets), the scheme $C_{L}(\Xi)$ is defined as expected by an equation whose square is an equation for the scheme $C_{L}(\widetilde{\Theta}) \cap T_{L}(P)$, and we say that "RST holds" at $L$. The main theorem of S-V5] is that the second case occurs (that is, the containment $C_{L}(\widetilde{\Theta}) \supset T_{L}(P)$ holds, so that $\left.\varphi_{*}\left(N_{|L|}(X)\right)=T_{L}(P)\right)$ if and only if $L$ is a very exceptional point of $\Xi$ (as defined in 2.1.2). 
4.2. Stable double points. In general, the most common singularities of Prym theta divisors are stable double points. More precisely, on all Pryms $P$ of dimension $\geq 6$ the stable singular locus is nonempty, of codimension $\leq 6$, and for general such $P$ the general singularity is a stable double point of $\operatorname{rank} 6$. If $\operatorname{dim}(P) \geq 7$ and the associated doubly covered curve $C$ has Clifford index $\geq 3$, the general stable singularity is a double point of rank $r$ where $3 \leq r \leq 6$, and if also $\operatorname{dim}(P) \geq 10$, all components of singularities of codimension $\leq 6$ are stable, and the generic stable singularity is not exceptional. References for these results can be found in [S-V4, §3.2]. Stable double points also occur, more specially, on lower dimensional Pryms.

We want to examine the relation between the rank of the quadric tangent cone at a stable double point and the infinitesimal structure of the Abel Prym map. In particular we will describe in some detail the parametrization $C_{|L|}(X) \rightarrow C_{L}(\Xi)$ of the tangent cone to $\Xi$, at a stable double point $L$. There are several possibilities according to the rank of the tangent quadric $Q \subset T_{L}(P)$ to $\Xi$ at $L$ and the rank of the map $M_{1}: T_{L}(P) \rightarrow \operatorname{Alt}(4) \cong \mathbb{C}^{6}$, defined by Mumford's skew symmetric matrix.

Since $\operatorname{rank}(Q) \leq \operatorname{rank}\left(M_{1}\right) \leq 6$, and the tangent quadric at a stable double point contains the Prym canonical curve, we have $3 \leq r k(Q) \leq 6$. The possibilities for the parametrization are as follows.

Theorem 1. Let $L$ be a stable double point of a Prym theta divisor $\Xi$, with tangent quadric $Q$.

1) Assume $r k(Q)=5$ or 6 . Then $r k(Q)=r k\left(M_{1}\right), L$ is not exceptional, and hence $C_{|L|}(X)=N_{|L|}(X)$ is an irreducible vector bundle parametrizing $C_{L}(\Xi)$. The restriction of the bundle over a general $\mathbb{P}^{2}$ in $|L|$ maps birationally onto $C_{L}(\Xi)$.

2) Assume $r k(Q)=4$. Then $r k\left(M_{1}\right)=$ either 4 or 5 .

(i) If $r k\left(M_{1}\right)=5,|L|$ contains exactly one exceptional pencil and no other singularities (example 6.4).

(ii) If $r k\left(M_{1}\right)=4,|L|$ contains exactly two exceptional pencils and no other singularities (example 6.2.1).

In both cases $C_{|L|}(X)=N_{|L|}(X)$ is irreducible, and the restriction of the parametrization $C_{|L|}(X) \rightarrow C_{L}(\Xi)$ to the fibers over each exceptional pencil is birational.

3) Assume $r k(Q)=3$. Then $r k\left(M_{1}\right)=$ either 3 or 4 .

(i) If $r k\left(M_{1}\right)=4$, then $|L|$ contains exactly one exceptional pencil and no other singularities; $C_{|L|}(X)=N_{|L|}(X)$ is irreducible, and the restriction of the parametrization $C_{|L|}(X) \rightarrow C_{L}(\Xi)$ to the fibers over that pencil is birational (example not included; should occur for a trigonal genus 6 curve whose Prym is a generic genus 5 Jacobian with a vanishing theta null).

(ii) If $r k\left(M_{1}\right)=3$, then $|L|$ contains a pencil of exceptional pencils, sweeping out a rank 4 quadric of singularities in $|L|$. In this case $C_{|L|}(X)=N_{|L|}(X)$ has 2 components, one supported over $|L|$ and one over the rank 4 quadric of singularities; only the second component dominates $C_{L}(\Xi)$. This second component is a bundle over the rank 4 quadric and its restriction to one of the exceptional lines maps birationally to $C_{L}(\Xi)$ (example 7).

Proof. Let $E \subset A l t(4)$ be the image of the linear map $M_{1}$, so that everything pulls back to $T_{L}(P)$ by the linear submersion $T_{L}(P) \rightarrow E$. That is, $N_{|L|}(X)$ and its map to $T_{L}(P)$ are induced from $\left.\mathbb{K}\right|_{E}$ and its map to $E$. In particular, $Q \subset T_{L}(P)$ is pulled back from $\Sigma \cap E$ which is defined by $q_{E}=\left.P f\right|_{E}$, the restriction of the Pfaffian 
quadratic form to $E$; so the rank of the tangent quadric $Q$ at $L$ is the same as the rank of $q_{E} \cdot N_{|L|}(X)$ will be irreducible if and only if $\left.\mathbb{K}\right|_{E}$ is, and whenever $N_{|L|}(X)$ is irreducible of dimension $p$ it must coincide with $C_{|L|}(X)$. Thus we analyze all the possibilities for a subspace $E \subset \operatorname{Alt}(4)$ with $3 \leq r k\left(q_{E}\right) \leq 6$ and find a "normal form" for each one.

For this purpose, with $V=H^{0}(L)$, consider the group homomorphism $G L(V) \rightarrow$ $G L\left(\Lambda^{2} V\right)$, defined by the natural action of the general linear group $G L(V)$ on the alternating 2-tensors. The famous "coincidence" (cf. [H, Lect. 22]) in this case of 4-dimensional $V$ is that $S L(V)$ maps onto the special orthogonal group of the rank 6 Pfaffian quadratic form. That is, in terms of a basis for $V$, the group homomorphism $S L(4, \mathbb{C}) \rightarrow S O(6, \mathbb{C})$ is surjective. One can deduce the surjectivity from general properties of algebraic groups (cf. [Bo, p. 88] as follows. The image of a homomorphism of algebraic groups is a closed subgroup of the target group and has dimension equal to the dimension of the source group minus the dimension of the kernel subgroup. In this case, it is easy to see directly that the kernel is finite (consisting of $\pm I$ in $\mathrm{SL}(4, \mathrm{C})$ ) and hence the image has dimension equal to $\operatorname{dim}(S L(4, \mathbb{C}))=15=\operatorname{dim}(S O(6, \mathbb{C}))$. Then, since the algebraic group $S O(6, \mathbb{C})$ is connected, hence irreducible, the image of the homomorphism must be all of $S O(6, \mathbb{C})$.

In case $E=A l t(4)$ we already have our normal form, as described in 4.1. Explicitly, we have the 6 -dimensional vector space of all $4 \times 4$ skew symmetric matrices:

$$
\left(\begin{array}{cccc}
0 & \lambda & x & y \\
-\lambda & 0 & y^{\prime} & z \\
-x & -y^{\prime} & 0 & \mu \\
-y & -z & -\mu & 0
\end{array}\right)
$$

with arbitrary numerical entries $\lambda, \mu, x, y, y^{\prime}, z \in \mathbb{C}$, and the Pfaffian is $\lambda \mu-$ $\left(x z-y y^{\prime}\right)$. Thus, in case of a surjection $T_{L}(P) \rightarrow \operatorname{Alt}(4)$ we can then view the entries $\lambda, \mu, x, y, y^{\prime}, z$ as linearly independent linear forms on $T_{L}(P)$, and the rank 6 Pfaffian quadratic form is given by the same formula.

We will mostly work projectively now, with quadric hypersurfaces of projective space. Notice first of all that for a smooth (equivalently, rank $r+1$ ) quadric hypersurface $\mathcal{R} \subset \mathbb{P}^{r}$, for $r \geq 2$, the special orthogonal group of $\mathcal{R}$, which we denote by $S O(r+1, \mathbb{C}) \subset S L(r+1, \mathbb{C})$, acts transitively on $\mathcal{R}$ and on the complement $\mathbb{P}^{r}-\mathcal{R}$, and hence (dually), $S O(r+1, \mathbb{C})$ acts transitively on $\{$ tangent hyperplanes of $\mathcal{R}\}$ and on $\{$ transverse hyperplanes of $\mathcal{R}\}$. Notice also that $S O(r+1, \mathbb{C})$ continues to act transitively on:

$\{$ smooth sections of $\mathcal{R}$ by the linear subspaces of any fixed dimension $\geq 2$ \}.

To see this, work step by step with transverse hyperplane sections, and observe that we can always lift an element of the special orthogonal group of a transverse hyperplane section by writing the ambient quadratic form $q$ in block form $q^{\prime}+x_{0}^{2}$ and using any given special orthogonal transformation in the $x^{\prime}$-coordinates and the identity on the $x_{0}$-coordinate to get a special orthogonal transformation in the full set of coordinates.

From now on in the proof, let $\mathcal{R} \subset \mathbb{P}^{5}$ be the smooth quadric hypersurface defined by the Pfaffian, and let $\mathbb{E}=\mathbb{P}(E)$ be the linear subspace of $\mathbb{P}^{5}$ obtained by projectivizing $E \subset \mathbb{C}^{6}$. It remains to consider the cases of $\operatorname{dim}(\mathbb{E})=4,3,2$, always under the assumption that the rank of the quadric hypersurface $\mathbb{E} \cap \mathcal{R}$ is at least 3 . 
- $\operatorname{dim}(\mathbb{E})=4$ : Then $\mathbb{E}$ is a hyperplane of $\mathbb{P}^{5}$, and there are 2 possibilities for the structure of the hyperplane section $\mathbb{E} \cap \mathcal{R}$ : either the hyperplane is transverse to $\mathcal{R}$ or the hyperplane is tangent to $\mathcal{R}$ at one point. The quadric hyperplane section $\mathbb{E} \cap \mathcal{R}$ has rank 5 in the first case, and has rank 4 in the second case. These are respectively cases 1 ) with rank 5 , and 2) (i) in the theorem. Each of these 2 cases forms one orbit under the action of $S O(6, \mathbb{C})$, and normal forms for these cases are given by the following kinds of skew symmetric matrices:

$$
\begin{aligned}
& \text { 1) with rank 5 : }\left(\begin{array}{cccc}
0 & \lambda & x & y \\
* & 0 & y^{\prime} & z \\
* & * & 0 & \lambda \\
* & * & * & 0
\end{array}\right) \text { or, if one prefers, }\left(\begin{array}{cccc}
0 & \lambda & x & y \\
* & 0 & y & z \\
* & * & 0 & \mu \\
* & * & * & 0
\end{array}\right), \\
& \text { and 2) (i) : }\left(\begin{array}{cccc}
0 & 0 & x & y \\
* & 0 & y^{\prime} & z \\
* & * & 0 & \mu \\
* & * & * & 0
\end{array}\right) .
\end{aligned}
$$

- $\operatorname{dim}(\mathbb{E})=3$ : Now the rank of $\mathbb{E} \cap \mathcal{R}$ can be 4 or 3 . If the rank is 4 , the codimension 2 section of $\mathcal{R}$ cut by $\mathbb{E}$ is tranvserse and there is one orbit of these, with normal form:

$$
\text { 2) (ii) : }\left(\begin{array}{llll}
0 & 0 & x & y \\
* & 0 & y^{\prime} & z \\
* & * & 0 & 0 \\
* & * & * & 0
\end{array}\right) \text {. }
$$

If the rank is 3 , then there is a unique singular point on $\mathbb{E} \cap \mathcal{R} \subset \mathbb{E} \cong \mathbb{P}^{3}$, and we can get to $\mathbb{E} \cap \mathcal{R}$ from $\mathcal{R}$ in 2 steps: first by taking a transverse hyperplane section of $\mathcal{R}$ and then by taking a tangent hyperplane section of the resulting smooth quadric in $\mathbb{P}^{4}$. Thus, there is one orbit of these and a normal form is:

$$
\text { 3) (i) : }\left(\begin{array}{cccc}
0 & 0 & x & y \\
* & 0 & y & z \\
* & * & 0 & \mu \\
* & * & * & 0
\end{array}\right) \text {. }
$$

- $\operatorname{dim}(\mathbb{E})=2$ : This case is quite easy since it is assumed that the conic $\mathbb{E} \cap \mathcal{R}$ has rank 3. Thus $\mathbb{E}$ is a projective plane transverse to the quadric $\mathcal{R} \subset \mathbb{P}^{5}$; there is one orbit of these under the action of $S O(6, \mathbb{C})$, and a normal form is given by the skew symmetric matrices:

$$
\text { 3) (ii) : }\left(\begin{array}{cccc}
0 & 0 & x & y \\
* & 0 & y & z \\
* & * & 0 & 0 \\
* & * & * & 0
\end{array}\right) \text {. }
$$

Now we identify all the positive dimensional isotropic subspaces of $|L| \cong \mathbb{P}^{3}$ in these cases. In other words, we want to find all vector subspaces of $V=H^{0}(L) \cong \mathbb{C}^{4}$ of dimension at least 2 that are isotropic w.r.t. $\beta$. Since a stable double point is not very exceptional, the dimension of any such isotropic vector subspace is exactly 2 .

If there exists a 2-dimensional isotropic subspace of $V$, then using a basis for it as the first two of four basis vectors for $V$ will make the $\lambda$-entry 0 in the skew symmetric matrix, and then the Pfaffian which reduces to $y y^{\prime}-x z$ will have rank $\leq 4$. 
Therefore, in case 1) $(r k(Q)=5$ or 6$)$ there are no isotropic subspaces of positive dimension.

Thus, if $A$ is the matrix of linear forms, we look for a nonzero vector $w$ such that the 4 entries in $A w$ span only a 2-dimensional subspace of linear forms. That is the condition for there to exist a vector $v$ linearly independent of $w$ for which $v^{t} A w=0$. Thus, if $w=\left(\begin{array}{l}a \\ b \\ c \\ d\end{array}\right) \in \mathbb{C}^{4}$, consider $A w=\left(\begin{array}{cccc}0 & 0 & x & y \\ * & 0 & y^{\prime} & z \\ * & * & 0 & \mu \\ * & * & * & 0\end{array}\right)\left(\begin{array}{l}a \\ b \\ c \\ d\end{array}\right)=$ $\left(\begin{array}{c}x c+y d \\ y^{\prime} c+z d \\ -x a-y^{\prime} b+\mu d \\ -y a-z b-\mu c\end{array}\right)$. Here we are not assuming at the moment that $x, y, y^{\prime}, z, \mu$ are linearly independent. In each of the remaining 4 cases we are given a specific basis of linear forms; namely, for 2) (i) we have all 5 ; for 2) (ii) $\mu=0$; for 3) (i) $y^{\prime}=y$; and for 3 ) (ii) $\mu=0$ and $y^{\prime}=y$.

In each case one can write out the 4 linear forms that appear in $A w$ in terms of the basis of linear forms for that case. In this way, one gets in each case a $4 \times 4$ numerical matrix $B$ depending on the entries of $w$, and the condition for $w$ to lie in a 2-dimensional isotropic subspace is that $r k(B) \leq 2$. (In other words, that $\operatorname{ker}(B) \subset \mathbb{C}^{4}$ have dimension $\geq 2$; intrinsically, the condition is that $\beta(-, w): V \rightarrow$ $T_{L}^{*}(P), v \mapsto \beta(v, w)$ have kernel of dimension at least 2.)

Consider case 2) (ii). Then, in the basis $\left\{x, y, y^{\prime}, z\right\}$ for this case,

$$
B=\left(\begin{array}{cccc}
c & d & 0 & 0 \\
0 & 0 & c & d \\
-a & 0 & -b & 0 \\
0 & -a & 0 & -b
\end{array}\right)
$$

and it is rather easy to see that the rank of this matrix is $\leq 2$ if and only if either $c, d=0$ or $a, b=0$. So the nonzero vector $w$ is in a 2-dimensional isotropic subspace iff $w$ has either the form $\left(\begin{array}{l}0 \\ 0 \\ c \\ d\end{array}\right)$ or $\left(\begin{array}{l}a \\ b \\ 0 \\ 0\end{array}\right)$.

In case 2) (i), with basis $x, y, y^{\prime}, z, \mu$, we get

$$
B=\left(\begin{array}{ccccc}
c & d & 0 & 0 & 0 \\
0 & 0 & c & d & 0 \\
-a & 0 & -b & 0 & d \\
0 & -a & 0 & -b & -c
\end{array}\right) \text { with rank } \leq 2 \text { iff } w \text { has the form }\left(\begin{array}{l}
a \\
b \\
0 \\
0
\end{array}\right)
$$

In case 3) (i), with basis $x, y, z, \mu$, we get

$$
B=\left(\begin{array}{cccc}
c & d & 0 & 0 \\
0 & c & d & 0 \\
-a & -b & 0 & d \\
0 & -a & -b & -c
\end{array}\right) \text { with rank } \leq 2 \text { iff } w \text { has the form }\left(\begin{array}{l}
a \\
b \\
0 \\
0
\end{array}\right)
$$


In case 3) (ii), with basis $x, y, z$, we get

$$
B=\left(\begin{array}{ccc}
c & d & 0 \\
0 & c & d \\
-a & -b & 0 \\
0 & -a & -b
\end{array}\right) \text { and the situation is more interesting. }
$$

Now the condition for $r k(B) \leq 2$ is that $(a, b)$ and $(c, d)$ be linearly dependent. In other words, we get an irreducible 1-dimensional family of isotropic 2-dimensional subspaces, including the 2 obvious ones $\{(*, *, 0,0)\}$ and $\{(0,0, *, *)\}$.

Finally, notice that as long as $\operatorname{dim}(E) \geq 4$ and $r k\left(q_{E}\right) \geq 3$, the total space $\left.\mathbb{K}\right|_{E}$ will be irreducible of dimension equal to $\operatorname{dim}(E)$. Indeed, the conical affine quadric hypersurface $\Sigma \cap E$ is irreducible and the map $\left.\mathbb{K}\right|_{E} \rightarrow \Sigma \cap E$ is a $\mathbb{P}^{1}$-bundle except over $0 \in \Sigma \cap E$, where the fiber is $\mathbb{P}^{3}$. But when $E$ has codimension $\leq 2$ in $\operatorname{Alt}(4)$, $\left.\mathbb{K}\right|_{E}$ is defined in the smooth 6 -dimensional variety $\mathbb{K}$ by at most 2 equations, and so every irreducible component of $\left.\mathbb{K}\right|_{E}$ must have dimension at least 4 .

But, as seen in the statement of Theorem 1, there is a surprise in the special case of 3) (ii). Namely, over the 3-dimensional vector space $E \subset A l t(4)$, the total space $\left.\mathbb{K}\right|_{E}$ becomes reducible since the fiber over $0 \in E$ (being $\mathbb{P}^{3}$ ) is already 3 -dimensional, and there is also a 3-dimensional irreducible component that dominates $\Sigma \cap E$, the conical affine quadric surface with standard equation $x z-y^{2}=0$. Thus $\left.\mathbb{K}\right|_{E}$ has pure dimension $3=\operatorname{dim}(E)$ in this case, with 2 irreducible components. Therefore $N_{|L|}(X)$ has pure dimension $p$ with 2 irreducible components, one containing $|L| \times \operatorname{ker}\left(M_{1}\right)$, and hence dominating $|L|$, and the other one not dominating $|L|$. Since $C_{|L|}(X)$ contains the irreducible component of $N_{|L|}(X)$ that dominates $|L|$ and this component of $C_{|L|}(X)$ does not map surjectively to the tangent cone $C_{L}(\Xi)$, the other $p$-dimensional component of $N_{|L|}(X)$ must also be part of $C_{|L|}(X)$. Thus $C_{L}(\Xi)$ and $N_{|L|}(X)$ still coincide in this special case.

Corollary (cf. [M, Lemma, p. 345]). Let $(P, \Xi)$ be the Prym variety of a connected étale double cover of a smooth nonhyperelliptic curve $C$ of genus $g \geq 3$, and set $p=g-1$. Then for any irreducible component $Z$ of $\operatorname{Sing}(\Xi)$ of dimension $\geq p-4$, every point $L \in Z$ is exceptional.

Proof. We will use the following two results.

(1) If $Z$ is any irreducible component of $\operatorname{Sing}(\Xi)$ with $h^{0}(L) \geq 4$ for all $L \in Z$, then there exists $L_{0} \in Z$ with $h^{0}\left(L_{0}\right)=4$, and hence $\left\{L \in Z \mid h^{0}(L)=4\right\}$ is open dense in $Z$.

(2) For any $L \in \Xi$, if $L$ is not exceptional, then $\operatorname{mult}_{L}(\Xi)=\frac{1}{2} h^{0}(L)$.

Now we prove the Corollary, with $Z$ as in the statement. It suffices to show that a general point of $Z$ is exceptional since being exceptional is a closed condition on points of $\Xi$. If a general point $L$ of $Z \subset \operatorname{Sing}(\Xi)$ has $h^{0}=2$, then $L$ is exceptional by (2). Otherwise, if every point of $Z$ has $h^{0} \geq 4$, then by (1) the general point has $h^{0}=4$. Then let $L \in Z$ be any point with $h^{0}(L)=4$. If $\operatorname{mult}_{L}(\Xi)>2$, then $L$ is exceptional by (2). So suppose that mult $_{L}(\Xi)=2$. Then stable double points are open dense in $Z$, and hence form an irreducible locus of dimension $\geq p-4$. Then since $L$ lies on a double point locus of dimension $\geq p-4, r k(Q) \leq 4$, so $L$ is exceptional by Theorem 1 .

Proof of (1): If $W \subset Z$ is an irreducible locus such that the general point $L \in W$ has $h^{0}(L)=k \geq 6$, then $\widetilde{W}=\left\{L\left(p^{\prime}-p+q^{\prime}-q\right) \mid L \in W\right.$ and $\left.p, q \in \widetilde{C}\right\}$ is an 
irreducible locus containing $W$ such that the general point $L \in \widetilde{W}$ has $h^{0}(L)=k-2$. Namely, $\widetilde{W}$ is irreducible since it is the image of $W \times \widetilde{C}^{2}$ and contains $W$ as the image of $W \times$ antidiagonal. Additionally, by [M] p. 345, l. 9] (cf. [S-V6, p. 233]) if $h^{0}(L)=k$ and $p$ is not a base point of $|L|$, then $h^{0}\left(L\left(p^{\prime}-p\right)\right)=k-1$, and if $q$ is not a base point of $\left|L\left(p^{\prime}-p\right)\right|$, then $h^{0}\left(L\left(p^{\prime}-p+q^{\prime}-q\right)\right)=k-2$.

Proof of (2): This is the Riemann singularities theorem for Prym theta divisors of [S-V3, Thm. 2.1, p. 486].

Although our proof of the Corollary runs along lines that are quite similar to those of [M], we hope that our presentation has clarified the role of the various main ingredients. In particular, if $s \wedge t$ is the decomposable element of $\Lambda^{2} H^{0}(L)$ that appears in Mumford's proof, then if $s, t$ are the first 2 vectors in a basis for $H^{0}(L)$, the upper left $2 \times 2$ block in the skew symmetric matrix will be 0 . When the base curve $C$ of the double cover is hyperelliptic, as is allowed in the Lemma of $[\mathbf{M}$, the singular locus of $\Xi$ is known explicitly, and it seems possible to verify directly that all the singular points of the Prym theta divisor are exceptional in that case.

\subsection{Questions and remarks.}

Question. Which skew-symmetric $n \times n$ matrices of linear forms can occur?

Remark. Those that actually occur as " $M_{1}$ " for an étale double cover of curves and $L$ on $\Xi$ are not arbitrary, since they satisfy at least the Isotropy Property of 2.1.4. For instance, the matrix

$$
\left(\begin{array}{cccc}
0 & 0 & 0 & x \\
0 & 0 & 0 & x \\
0 & 0 & 0 & x \\
-x & -x & -x & 0
\end{array}\right)
$$

cannot occur since the 3 -dimensional maximal isotropic subspace $V_{1}=\{(*, *, *, 0)\}$ does not contain the 2 -dimensional isotropic subspace $V_{2}$ spanned by $(1,-1,0,0)$ and $(0,0,0,1)$, yet $V_{1}$ and $V_{2}$ have the nontrivial vector $(1,-1,0,0)$ in common.

For the Jacobian of a curve, the matrix of linear forms representing a cup product bilinear map

$$
H^{0}(L) \times H^{0}\left(\Omega \otimes L^{*}\right) \rightarrow H^{0}(\Omega)=\{\text { linear forms on the tangent space }\}
$$

satisfies a very strong "regularity property" (exploited by Kempf; cf. [K1, K2, K3] ) since for any nonzero vector in one of the factors, the induced linear map on the other factor is injective.

Question. For $L \in \Xi$, what are the possible subvarieties of $|L| \subset X$ over which the irreducible components of the normal space $N_{|L|}(X)$ of $X$ along $|L|$ are supported?

Remarks. (i) We do not know whether there can be an irreducible component of the normal space $N_{|L|}(X)$ that does not contain an irreducible component of the normal cone $C_{|L|}(X)$. In case (1) of 3.1, i.e. if $L \in \Xi$ is exceptional but not very exceptional, we do not know whether there can be an irreducible component of $N_{|L|}(X)$ of dimension $>p$.

(ii) Let us record the most elementary dimension estimate to ensure both irreducibility of the normal space $N_{|L|}(X)$ and set-theoretic equality of $C_{|L|}(X)$ and $N_{|L|}(X)$, hence irreducibility of the normal cone $C_{|L|}(X)$. The dimension of the normal vector spaces, i.e. the fibers of $N_{|L|}(X) \rightarrow|L|$, can vary over $|L|$ since it goes up at any exceptional divisor $D$ in $|L|$. Consider the (locally closed) locus 
$I_{k}(L)$ consisting of all divisors $D \in|L|$ for which the maximal isotropic subspace of $\beta$ through $D$ has dimension exactly $k-1$ (as projective linear subspace of $|L|$ ). Then for $D \in I_{k}(L)$, we know [S-V3] that $\operatorname{dim}\left(T_{D}(X)\right)=p+(k-1)$. Therefore the fiber of the normal space $N_{|L|}(X)$ over such a point $D$ has dimension $p+(k-1)-(n-1)=p-(n-k)$. Since the normal cone $C_{|L|}(X)$ has pure dimension $p$ and the fibers of the normal cone are contained in the fibers of the normal space, it follows that as long as $\operatorname{dim}\left(I_{k}(L)\right)<n-k$ there cannot be an irreducible component of the normal cone that dominates an irreducible component of $I_{k}(L)$ (i.e. that lies over an irreducible component $Z$ of the closure of $I_{k}(L)$ and maps onto $Z$ ). In fact since all components of $N_{|L|}(X)$ have dimension $\geq p$, there is also no component of the normal space dominating $I_{k}(L)$ when $\operatorname{dim}\left(I_{k}(L)\right)<n-k$. For instance, taking $k=2$, unless there are at least $\infty^{n-3}$ exceptional pencils in $|L|$ there is not a component of $C_{|L|}(X)$ dominating a component of $I_{2}(L) \subset|L|$. Thus if for all $k$ with $2 \leq k \leq n$, we have $\operatorname{dim}\left(I_{k}(L)\right)<n-k$, then the normal cone $C_{|L|}(X)$ is irreducible and equals the normal space $N_{|L|}(X)$.

\section{General structure of the very exceptional Case}

As before, let $(P, \Xi)$ be the Prym variety of a connected étale double cover $\pi: \widetilde{C} \rightarrow C$ of a nonhyperelliptic smooth curve of genus $g \geq 3$. Thus $p=\operatorname{dim}(P)=$ $g-1$ and for any point $L \in \Xi$, the tangent cone $C_{L}(\Xi) \subset T_{L}(P)$ is a purely $p-1$ dimensional conical affine subvariety of the $p$ dimensional vector space $T_{L}(P) \cong$ $T_{0}(P)$. Now let $L$ on $\Xi$ be a very exceptional or "Shokurov" singularity on $\Xi$; i.e. assume there exists a subspace of $H^{0}(L)$ which has dimension more than $\frac{1}{2} h^{0}(L)$ and which is isotropic for the Mumford pairing $\beta: H^{0}(L) \times H^{0}(L) \rightarrow H^{0}\left(\Omega_{C}(\eta)\right)$. If it is maximal isotropic, then it has form $\pi^{*}\left(H^{0}(M)\right) \cdot u$, where $M$ is the unique Shokurov line bundle on $C$ for $L$, and $u$ defines a divisor $B$ on $\widetilde{C}$ with no invariant part [S-V5].

Theorem 2. Let $L \in \Xi$ be a very exceptional singularity and let $\pi^{*}\left(H^{0}(M)\right) \cdot u \subset$ $H^{0}(L)$ be the unique maximal isotropic subspace of dimension $h^{0}(M)>\frac{1}{2} h^{0}(L)$. Then the tangent cone $C_{L}(\Xi)$ is the image of those irreducible components of the normal cone $C_{|L|}(X)$ that lie over $\pi^{*}|M|+B \subset|L|$. More precisely, the union, over all divisors in $|L|-\left(\pi^{*}|M|+B\right)$, of the images in $T_{L}(P)$ of the differential of the Abel Prym map $\varphi: X \rightarrow \Xi$ cannot have dimension more than $p-3$. In particular this union cannot contain a $p-1$ dimensional component of the tangent cone $C_{L}(\Xi)$ of $\Xi$ at $L$.

Proof. We may as well assume that the subspace $\pi^{*} H^{0}(M) \cdot u$ has dimension $h^{0}(M)$ strictly between $\frac{1}{2} h^{0}(L)$ and $h^{0}(L)$, since otherwise $|L|-\left(\pi^{*}|M|+B\right)$ is empty and the result holds vacuously. Let $s$ be any element of $H^{0}(L)-\pi^{*} H^{0}(M) \cdot u$. Recall from the Proposition in 2.2.1 that if $D_{s}$ is the divisor defined in $\widetilde{C}$ by $s$, then $\varphi_{*}\left(T_{D_{s}}(X)\right)=\operatorname{Im}(\beta(s,-))^{\perp}$. In particular, the containment $\varphi_{*}\left(T_{D_{s}}(X)\right) \subset$ $\beta\left(s, \pi^{*} H^{0}(M) \cdot u\right)^{\perp}$ holds.

Now we claim that the restriction of $\beta(s,-): H^{0}(L) \rightarrow H^{0}\left(\Omega_{C}(\eta)\right)$ to the subspace $\pi^{*} H^{0}(M) \cdot u \subset H^{0}(L)$ is injective, and consequently

$$
\operatorname{dim}\left(\varphi_{*}\left(T_{D_{s}} X\right)\right) \leq p-h^{0}(M) .
$$


Indeed, if $\beta(s, \sigma)=0$ for some nonzero $\sigma \in \pi^{*} H^{0}(M) \cdot u$, then the maximal isotropic subspace containing $s$ would intersect the isotropic subspace $\pi^{*} H^{0}(M) \cdot u$ nontrivially. Hence by the properties of $\beta$ summarized in 2.1.4, the span of $s$ and $\pi^{*} H^{0}(M) \cdot u$ would be isotropic, contradicting the maximality of $\pi^{*} H^{0}(M) \cdot u$ as an isotropic subspace of $H^{0}(L)$.

Moreover, for any $t \in \pi^{*} H^{0}(M) \cdot u$, we have

$$
\beta\left(s+t, \pi^{*} H^{0}(M) \cdot u\right)=\beta\left(s, \pi^{*} H^{0}(M) \cdot u\right),
$$

so that $\varphi_{*}\left(T_{D_{s+t}}(X)\right) \subset \beta\left(s, \pi^{*} H^{0}(M) \cdot u\right)^{\perp}$ as well. From this we obtain a dimension count, that is,

$$
\begin{aligned}
& \operatorname{dim}\left(\bigcup_{s \in H^{0}(L)-\pi^{*}\left(H^{0}(M)\right) \cdot u} \varphi_{*}\left(T_{D_{s}}(X)\right)\right) \\
\leq & \operatorname{dim}\left(\mathbb{P}\left(H^{0}(L) / \pi^{*}\left(H^{0}(M)\right) \cdot u\right)\right)+\left(p-h^{0}(M)\right) \\
= & \left(h^{0}(L)-h^{0}(M)-1\right)+\left(p-h^{0}(M)\right)=p+\left(h^{0}(L)-2 h^{0}(M)\right)-1 .
\end{aligned}
$$

But $h^{0}(L)-2 h^{0}(M) \leq-2$ since it is an even integer and is negative for very exceptional $L$, so the union has dimension $\leq p-3$. Therefore, the unique irreducible component of the normal cone $C_{|L|}(X)$ supported over all of $|L|$ cannot map onto an irreducible component of the tangent cone $C_{L}(\Xi)$. It follows that the union of the irreducible components of $C_{|L|}(X)$ whose support is contained in the subspace $\pi^{*}|M|+B$ of very exceptional divisors must map onto $C_{L}(\Xi)$.

\section{EXAMPles of THE EXPECTED TYPICAL STRUCTURE}

6.1. Abel Prym differential for the Prym representation of a cubic threefold $\mathbf{W}$ defined by a line $\ell$ in $\mathbf{W}$. We will compute the parametrization $\mathbb{P} C_{|L|}(X)$ $\rightarrow \mathbb{P} C_{L}(\Xi)$ for the beautiful case of a Prym representation of the cubic threefold, in which the unique singularity is very exceptional.

Let $\varphi: X \rightarrow P$ be the Abel Prym map for an odd double cover $\pi: \widetilde{C} \rightarrow C$ of a plane quintic curve $C$ associated to a general line $\ell$ in a smooth cubic threefold $W$. Then the polarized Prym $P$ is isomorphic to the polarized intermediate Jacobian of $W$. In particular $X$ maps onto the theta divisor $\Xi$, a fourfold in $P$ with one singular point $L$, over which the fiber of $\varphi$ is $E=\left|g_{10}^{3}\right| \cong\left|\pi^{*}\left(g_{5}^{2}\right)\right| \cong \mathbb{P}^{3}$, and the projective tangent cone to $\Xi$ at $L$ is $W$. A clear and complete proof of this story on the singularities of theta for the intermediate Jacobian of a cubic threefold was published by Beauville [B3]; the statement is in Mumford's paper [M, p. 348]. Some additional references are [F, p. 80], [S-V1, Ex. 6.4, p. 670], and [S-V3 §5, pp. 503-506].

Theorem 3. If $W$ is a smooth cubic threefold, the Abel Prym map $\varphi: X \rightarrow \Xi$ defined by a general line $\ell$ in $W$ induces a map from the reducible (2 components) projective normal cone along $\left|\pi^{*}\left(g_{5}^{2}\right)\right|$ in $X$ onto the projective tangent cone (isomorphic to $W$ ) of $\Xi$ at $L=\pi^{*}\left(g_{5}^{2}\right)$. One component is a trivial $\mathbb{P}^{1}$ bundle over $|L| \cong \mathbb{P}^{3}$ which collapses onto the line $\ell$, and the fibers of the other component, supported over $\pi^{*}\left|g_{5}^{2}\right| \cong \mathbb{P}^{2}$, map isomorphically to the cubic surfaces cut on $W$ by the net of hyperplanes through $\ell$ in $\mathbb{P} T_{0}(P) \cong \mathbb{P}^{4}$.

Proof. The singular locus of $X$ consists precisely of the subvariety $S=\pi^{*}\left|g_{5}^{2}\right| \cong \mathbb{P}^{2}$ of $E$; cf. [S-V3, S-V5]. Indeed, $L=\pi^{*}\left(g_{5}^{2}\right)$ is the only singular point of $\Xi$, and 
$\pi^{*}\left|g_{5}^{2}\right|$ is the locus of (very) exceptional divisors in the 3-dimensional projective space $\left|\pi^{*}\left(g_{5}^{2}\right)\right|$; see 2.1.3. Moreover by the general theory of blowups, the projective normal cone of $X$ along $E$ maps surjectively onto $W$ by the restricted differential $\varphi_{*}$ of $\varphi$. (Indeed the differential $\varphi_{*}$ on the projective normal space $\mathbb{P} N_{E}(X)$ containing this normal cone $\mathbb{P} C_{E}(X)$ is induced by a map on $\mathbb{P} N_{E}\left(\widetilde{C}^{(10)}\right)$, from blowing up the ambient smooth variety $\widetilde{C}^{(10)}$ along $E$, and this latter map is the differential of the Abel map on $\widetilde{C}^{(10)}$.)

The Zariski tangent spaces of $X$ have dimension 5 away from $S$, and dimension 7 at points of $S$, according to [S-V3, Cor. 2.14, p. 491]. The fibers of $\varphi$ are all smooth by the Riemann-Kempf, Mattuck-Mayer theorem, and since $E$ is the only fiber which is not isomorphic to $\mathbb{P}^{1}$, the kernel of the differential of $\varphi$ acting on the Zariski tangent space to $X$ has dimension 1 away from $E$ and dimension 3 at points of $E$. Thus the image of the differential of $\varphi$ has linear dimension $5-1=4$ away from $E, 5-3=2$ at points of $E-S$, and $7-3=4$ at points of $S$.

We claim the image space of that differential is constant on $E-S$; i.e. at all points of $E-S$ the image of the differential of $\varphi$ is the same 2-dimensional linear subspace of the tangent space to $P$ at $L$. Equivalently the projective image of the differential of $\varphi$ is the same projective line in $W$ for every point of $E-S$. To see this, note that the differential of $\varphi$ defines a morphism from $E-S$ to the Fano surface of lines in $W$. Since this Fano surface embeds in the intermediate Jacobian of $W$ it does not contain any rational curves. Since $E-S$ is rationally connected, this morphism is constant. (The constancy of the map on $E-S$ in this case is also an instance of Theorem 2 in section 5.)

Thus the 4-dimensional irreducible component of the projective normal cone of $X$ along $E$ corresponding to the closure of the normal $\mathbb{P}^{1}$ bundle of $E-S$ maps by the differential of $\varphi$ onto a single line in $W$. Since the full normal cone maps onto $W$ there must be other components supported in $S$. We claim there is only one, supported on all of $S$.

First of all, at points of $S$ the Zariski tangent space to $X$ has dimension 7 and the kernel of $\phi_{*}$ has dimension 3, so the Zariski normal spaces have dimension 4 . Hence each fiber of the projective normal cone at a point of $S$ is contained in a $\mathbb{P}^{3}$, and $\phi_{*}$ is injective on this $\mathbb{P}^{3}$. Since $W$ does not contain any copies of $\mathbb{P}^{3}$, the fibers of the projective normal cone at points of $S$ have dimension at most 2 . In particular, since $S \cong \mathbb{P}^{2}$, no component of the pure 4-dimensional projective normal cone is supported in a proper subvariety of $S$.

Thus if $Z$ is the part of the projective normal cone supported in $S$, then every component of $Z$ is supported on all of $S$, all fibers of $Z \rightarrow S$ have pure dimension 2 , and each fiber injects by $\phi_{*}$ into the cubic surface cut from $W$ by the image of the projective normal space under $\phi_{*}$, which is isomorphic to $\mathbb{P}^{3}$. Since $W$ contains no 2-planes, each of these cubic surface sections is irreducible. Hence each fiber of $Z \rightarrow S$ is an irreducible cubic surface, and by [Shaf, Ch. I, 6.3, Theorem 8, p. 77] $Z$ itself is irreducible; i.e. there is only one irreducible component of the normal cone supported on $S$.

Thus the projective normal cone to $E$ in $X$ is pure 4-dimensional and has two irreducible components. One component is supported on $E \cong \mathbb{P}^{3}$ and is at least generically a $\mathbb{P}^{1}$ bundle that maps onto a unique line $\ell^{\prime}$ in $W$. The other component is supported on $\pi^{*}\left|g_{5}^{2}\right| \cong \mathbb{P}^{2}$ and maps onto $W$, with each fiber of this component over a point of $\pi^{*}\left|g_{5}^{2}\right|$ mapping isomorphically onto a cubic surface hyperplane 
section of $W$. We will show that the component of the projective normal cone in $X$ along $\pi^{*}\left|g_{5}^{2}\right|$ maps by the differential of $\varphi$ to the net of cubic surface sections of $W$ cut by the net of $\mathbb{P}^{3}$ 's passing through the line $\ell$, and in particular that $\ell=\ell^{\prime}$.

To see that $\ell^{\prime}=\ell$, we recall the way the line $\ell$ is used to construct the curve $C$. First $W$ in $\mathbb{P}^{4}$ is fibered over the $\mathbb{P}^{2}$ of planes through $\ell$, with fibers the net of conics residual to $\ell$ in each plane section of $W$. Then $C$ is the curve of reducible conics in this net, and $\widetilde{C}$ is the curve of components of reducible conics. The Prym canonical model of $C$ in $\mathbb{P}^{4}$ is the locus of singular points of reducible conics and is mapped isomorphically to the plane quintic model by the projection $\mathbb{P}^{4} \rightarrow \mathbb{P}^{2}$ with the line $\ell$ as center.

Under this projection, a line in $\mathbb{P}^{2}$ corresponds to a hyperplane in $\mathbb{P}^{4}$ containing $\ell$. Hence each divisor of $\left|g_{5}^{2}\right|=|M|$ cut on the quintic by a line in $\mathbb{P}^{2}$ corresponds to the moving part of the Prym canonical divisor cut on the Prym canonical model by the corresponding hyperplane in $\mathbb{P}^{4}$ through $\ell$. Since by Riemann-Roch only one Prym canonical divisor vanishes on each divisor of the $g_{5}^{2}$, the net of divisors of the $g_{5}^{2}$ on the Prym canonical curve span the net of hyperplanes in $\mathbb{P}^{4}$ through $\ell$. Moreover, recalling that $K+\eta=M+(M+\eta)$, the fixed part of the corresponding net of Prym canonical divisors, i.e. the unique divisor in $|M+\eta|$, spans the line $\ell$.

If $D$ is a divisor of $|M|=\left|g_{5}^{2}\right|$, and $\pi^{*}(D)$ is the corresponding very exceptional divisor in $|L|$, it follows from the geometric description of the Prym canonical map ([S-V3, Lemma 5.2, p. 504] or [T] ) that the hyperplane spanned by $D$ on the Prym canonical curve is contained in the intersection of $\mathbb{P} T_{0}(P)$ with the span of the divisor $\pi^{*}(D)$ on the canonical model of $\widetilde{C}$ in $\mathbb{P} T_{0}(\tilde{J})$. Moreover by the formula in $[\mathrm{S}-\mathrm{V3}$, Lemma 2.6, p. 488] this intersection equals the (projective) image of the differential of $\varphi$.

It follows that the base locus $\ell$ of the hyperplanes spanned by divisors of $|M|$ on the Prym canonical curve must equal the base locus $\ell^{\prime}$ of the images of $\varphi_{*}$ at points of $S=\pi^{*}|M|$ in $X$. One can also compute directly the image of $\varphi_{*}$ at the unique divisor of $\pi^{*}|M+\eta|$ in $E-S$. Namely by the same argument as above, this image contains and hence equals the span $\ell$ of the divisor $|M+\eta|$ on the Prym canonical curve. Since $\varphi_{*}$ has the same image $\ell^{\prime}$ at all points of $E-S$, we again see that $\ell=\ell^{\prime}$.

Remarks. This discussion of the parametrization $\mathbb{P} C_{E}(X) \rightarrow \mathbb{P} C_{\left\{\pi^{*}\left(g_{5}^{2}\right)\right\}}(\Xi)$ associated to a cubic threefold $W \subset \mathbb{P}^{4}$ and general line $\ell$ on it shows that the normal cone $C_{E}(X)$ with its reduced structure has multiplicity 4 at a general point of $S=\pi^{*}\left|g_{5}^{2}\right|$. The irreducible component of $C_{E}(X)$ supported over $S$ is the family of affine cubic cones over the hyperplane sections of $W$ through $\ell$, and the component supported over all of $E$ is smooth in this case. Indeed, with $\mathbb{P} C_{E}(X) \hookrightarrow E \times \mathbb{P}^{4}$, it was shown that the irreducible component that dominates $E$ maps onto a line $\ell^{\prime} \subset \mathbb{P}^{4}$ and hence is a closed subvariety of the 4 -dimensional variety $E \times \ell^{\prime}$. Then, since $\mathbb{P} C_{E}(X)$ has pure dimension $=\operatorname{dim}(X)-1=4$, this component must be (set-theoretically) equal to $E \times \ell^{\prime}$.

For the line bundle $L=\pi^{*}\left(g_{5}^{2}\right)$ in this case, Mumford's skew-symmetric matrix of linear forms is $4 \times 4$ and can be written (see [Shok, p. 121]) with a $3 \times 3$ upper left block of 0 's and 3 linearly independent linear forms in the 4 th column (above the 0 in the bottom right corner). The 3 linear forms give equations for the hyperplanes in $\mathbb{P}^{4}$ corresponding to the pullback of sections of $g_{5}^{2}$ on $\mathbb{P}^{2}$, hence define the line $\ell \subset \mathbb{P}^{4}$. This base locus also equals $\ell^{\prime}$ by Corollary 1 of section 4.1 . 


\subsection{Examples of nondegeneracy.}

6.2.1. Example (irreducible normal cone over an exceptional singularity). Consider a nonhyperelliptic smooth curve $C$ with distinct theta characteristics $M_{1}$ and $M_{2}$ such that $\left|M_{1}\right|$ and $\left|M_{2}\right|$ are base point free pencils. Let $\eta=M_{1} \otimes M_{2}^{*}$ and let $\pi: \widetilde{C} \rightarrow C$ be the corresponding étale double cover. Then the line bundle $L=\pi^{*}\left(M_{1}\right)=\pi^{*}\left(M_{2}\right)$ on $\widetilde{C}$ defines an exceptional singularity of the Prym theta divisor $\Xi \subset P$. $H^{0}(L) \cong H^{0}\left(M_{1}\right) \oplus H^{0}\left(M_{2}\right)$ is 4-dimensional, and forming a basis for $H^{0}(L)$ by using a basis for each $H^{0}\left(M_{i}\right)$, Mumford's $4 \times 4$ skew-symmetric matrix of linear forms has a $2 \times 2$ block of 0 's in the upper left and lower right. Since the upper right $2 \times 2$ block is formed from the cup product $H^{0}\left(M_{1}\right) \times H^{0}\left(M_{2}\right) \rightarrow H^{0}\left(\Omega_{C}(\eta)\right)$, the linear forms appearing as the 4 entries there are linearly independent (since $\left|M_{1}\right|$ and $\left|M_{2}\right|$ are distinct base point free pencils). It follows that the only singular points in $|L| \cong \mathbb{P}^{3}$ of the divisor variety $X$ are the points of the 2 lines $\pi^{*}\left|M_{i}\right|$. By the elementary dimension estimate in the Remarks at the end of section 4 , the normal cone $C_{|L|}(X)$ is irreducible of dimension $p=\operatorname{dim}(P)$ and is set-theoretically equal to the normal space $N_{|L|}(X)$.

6.2.2. Example (variable image from the smooth points over a very exceptional singularity). We will construct a very exceptional singularity of numerical type $(4,6)$ to show that (in contrast to the $(n-1, n)$ case) it is possible for the image subspace of the Abel Prym differential on the fibers of $N_{|L|}(X)$ over the smooth points of $X$ in $|L|$ to vary in $T_{L}(P)$. For the example we will produce a nonhyperelliptic smooth curve $C$ having vanishing theta nulls $M_{1}, M_{2}$ such that $h^{0}\left(M_{1}\right)=4$ and $\left|M_{2}\right|$ is a base point free pencil. Then with $\eta=M_{1} \otimes M_{2}^{*}$ and $L=\pi^{*}\left(M_{1}\right)=\pi^{*}\left(M_{2}\right)$ on $\widetilde{C}$, we will get similarly to the previous example, a $6 \times 6$ skew-symmetric matrix of linear forms with a $4 \times 4$ block of 0 's in the upper left and a $2 \times 2$ block of 0 's in the lower right. We claim that the 8 linear forms appearing in the $4 \times 2$ upper right block are linearly independent.

Thus we need to check that we get maximal rank $(4 \cdot 2=8)$ for the cup product $H^{0}\left(M_{1}\right) \otimes H^{0}\left(M_{2}\right) \rightarrow H^{0}\left(M_{1} \otimes M_{2}\right)$, and we can use the "base-point-free pencil trick" to do so; cf. [ACGH, p. 126]. That is, starting with the base point free pencil $\left|M_{2}\right|$ on $C$, consider the resulting surjection $\mathcal{O}_{C} \otimes H^{0}\left(M_{2}\right) \rightarrow M_{2}$ from a trivial rank 2 vector bundle to the line bundle $M_{2}$. The kernel line bundle is isomorphic to $M_{2}^{*}$, so tensoring with the line bundle $M_{1}$ and taking global sections we get

$$
0 \rightarrow H^{0}\left(M_{1} \otimes M_{2}^{*}\right) \rightarrow H^{0}\left(M_{1}\right) \otimes H^{0}\left(M_{2}\right) \rightarrow H^{0}\left(M_{1} \otimes M_{2}\right) \rightarrow \ldots
$$

Hence if $M_{1} \not M_{2}$ has the same degree as $M_{2}$, then $H^{0}\left(M_{1} \otimes M_{2}^{*}\right)=0$ and the cup product linear map is injective.

One way to carry out the construction is as follows. Take any nonhyperelliptic smooth curve $C_{0}$ of genus 4 with a vanishing theta null $M$. Then we will choose an appropriate order 8 subgroup $G=\left\langle\eta_{0}, \alpha, \beta\right\rangle$ of $1 / 2$-periods on $C$ in order to carry out the construction. Namely, reducing an integral symplectic basis for $H_{1}(C, \mathbb{Z})$ modulo 2 to equate $H_{1}(C, \mathbb{Z} /(2)) \cong(\mathbb{Z} /(2))^{g} \times(\mathbb{Z} /(2))^{g}$ and express each $1 / 2$-period as two rows of 0 's and 1 's, let

$$
\eta_{0}=\left(\begin{array}{llll}
0 & 1 & 0 & 0 \\
0 & 0 & 0 & 0
\end{array}\right), \alpha=\left(\begin{array}{cccc}
0 & 0 & 1 & 0 \\
0 & 0 & 0 & 0
\end{array}\right), \beta=\left(\begin{array}{cccc}
1 & 0 & 0 & 0 \\
1 & 0 & 0 & 0
\end{array}\right) .
$$

Of course there do exist nonhyperelliptic smooth curves of genus 4 with a vanishing theta null. What is convenient is that for such a curve $C_{0}$ there is exactly 1 vanishing 
theta null $M$ (even theta characteristic with $h^{0}>0$ ), $|M|$ is a base point free pencil, and all the odd theta characteristics are nonsingular (i.e. $h^{0}=1$ ). Now consider the 8 distinct theta characteristics $\{M(\sigma) \mid \sigma \in G\}$. Four of them are even:

$$
M=\left[\begin{array}{llll}
0 & 0 & 0 & 0 \\
0 & 0 & 0 & 0
\end{array}\right],\left[\begin{array}{llll}
0 & 1 & 0 & 0 \\
0 & 0 & 0 & 0
\end{array}\right],\left[\begin{array}{llll}
0 & 0 & 1 & 0 \\
0 & 0 & 0 & 0
\end{array}\right],\left[\begin{array}{llll}
0 & 1 & 1 & 0 \\
0 & 0 & 0 & 0
\end{array}\right] ;
$$

and four of them are odd:

$$
A=\left[\begin{array}{llll}
1 & 0 & 0 & 0 \\
1 & 0 & 0 & 0
\end{array}\right],\left[\begin{array}{llll}
1 & 1 & 0 & 0 \\
1 & 0 & 0 & 0
\end{array}\right],\left[\begin{array}{llll}
1 & 0 & 1 & 0 \\
1 & 0 & 0 & 0
\end{array}\right],\left[\begin{array}{llll}
1 & 1 & 1 & 0 \\
1 & 0 & 0 & 0
\end{array}\right] .
$$

Now pass to the étale double cover $\pi_{0}: C_{1} \rightarrow C_{0}$ defined by $\eta_{0}$, and pull back $G$ and the theta characteristics $\{M(\sigma)\}$. Thus consider on $C_{1}$ the order 4 pulled-back group $\widetilde{G}$ of $1 / 2$-periods and the four pulled-back theta characteristics. Thus $\widetilde{G}$ is generated by $\eta_{1}=\pi_{0}^{*}(\alpha)$ and $\tilde{\beta}=\pi_{0}^{*}(\beta)$, and we label the theta characteristics $\widetilde{A}=\pi_{0}^{*}(A)$ and $\widetilde{M}=\pi_{0}^{*}(M)$.

Now we have

$$
h^{0}(\widetilde{A})=2, h^{0}\left(\widetilde{A}\left(\eta_{1}\right)\right)=2, h^{0}(\widetilde{M})=2, h^{0}\left(\widetilde{M}\left(\eta_{1}\right)\right)=0 .
$$

Next pass to the étale double cover $\pi_{1}: C=C_{2} \rightarrow C_{1}$ defined by $\eta_{1}$. Let $M_{1}=$ $\pi_{1}^{*}(\widetilde{A}), M_{2}=\pi_{1}^{*}(\widetilde{M})$, and let $\eta=\pi_{1}^{*}(\tilde{\beta})$. We now have $h^{0}\left(M_{1}\right)=4, h^{0}\left(M_{2}\right)=2$, and $M_{1}(\eta)=M_{2}$.

So finally for $\pi: \widetilde{C} \rightarrow C$ defined by $\eta$, the line bundle $L=\pi^{*}\left(M_{1}\right)$ on $\widetilde{C}$ has a $6 \times 6$ skew-symmetric matrix of linear forms with a $4 \times 4$ block of 0 's in the upper left, a $2 \times 2$ block of 0 's in the lower right, and 8 linearly independent linear forms in the $4 \times 2$ upper right block.

It remains to explain why it follows that the images vary over the smooth points. Choose a basis $\left\{s_{0}, \ldots, s_{3}\right\}$ for $H^{0}\left(C, M_{1}\right)$, and a basis $\left\{s_{4}, s_{5}\right\}$ of $H^{0}\left(C, M_{2}\right)$, and consider $\left\{s_{0}, \ldots, s_{5}\right\}$ as a basis for $H^{0}(\widetilde{C}, L)$ (identifying the sections with their pullbacks). Denote the corresponding coordinates on $|L| \cong \mathbb{P}^{5}$ by $z_{0}, \ldots, z_{5}$. Then $\pi^{*}\left(\left|M_{1}\right|\right)=\left\{z_{4}=z_{5}=0\right\} \cong \mathbb{P}^{3}$ is the largest linear subspace of $|L| \cap \operatorname{Sing}(X)$. Hence that intersection cannot be all of $\mathbb{P}^{5}$, and thus has dimension at most 4 . Then the smooth points of $X$ are dense in $|L|-\pi^{*}\left(\left|M_{1}\right|\right) \cong \mathbb{P}^{5}-\mathbb{P}^{3}=\left\{\left(z_{4}, z_{5}\right) \neq(0,0)\right\}$. In particular the projection $\mathbb{P}^{5}-\mathbb{P}^{3} \rightarrow \mathbb{P}^{1}$, taking $\left(z_{0}, \ldots, z_{5}\right)$ to $\left(z_{4}, z_{5}\right)$, is not constant on points of $|L|-\operatorname{Sing}(X)$.

If we denote the last two columns of the Mumford-Kempf matrix of linear forms by $v$ and $w$, then at every point of $|L|-\pi^{*}\left(\left|M_{1}\right|\right)$, the image of $\varphi_{*}$ has codimension at most 5 in $T_{L}(P)$ and is contained in the codimension 4 base locus of the entries of the column of linear forms: $z_{4} v+z_{5} w$. If $\left(z_{4}, z_{5}\right)$ and $\left(\tilde{z}_{4}, \tilde{z}_{5}\right)$ are not proportional, the intersection of the base loci of $z_{4} v+z_{5} w$ and of $\tilde{z}_{4} v+\tilde{z}_{5} w$ has codimension 8 , hence cannot contain the image of $\varphi_{*}$ at any point of $|L|-\pi^{*}\left(\left|M_{1}\right|\right)$.

Hence if $D, E$ are points of $|L|$ that are smooth on $X$ and have last two coordinates $\left(z_{4}, z_{5}\right)$ and $\left(\tilde{z}_{4}, \tilde{z}_{5}\right)$ that are not proportional, then the images of $\varphi_{*}$ at $D$ and $E$ are distinct. In fact a point of $|L|$ is smooth on $X$ if and only if the image of $\varphi_{*}$ has codimension 5 , so we can choose $D$ and $E$ as the points with coordinates $(1,0,0,0,0,1)$ and $(1,0,0,0,1,0)$ in $|L| \cong \mathbb{P}^{5}$. By similar reasoning one can see that the intersection $|L| \cap \operatorname{Sing}(X)$ consists of just two components, the union of $\pi^{*}\left(\left|M_{1}\right|\right):\left\{z_{4}=z_{5}=0\right\} \cong \mathbb{P}^{3}$ and $\pi^{*}\left(\left|M_{2}\right|\right):\left\{z_{0}=\cdots=z_{3}=0\right\} \cong \mathbb{P}^{1}$. 
6.3. Examples of totally exceptional pencils. When the Prym variety is the Jacobian of a curve, the tangent cone at any double point is then known to be irreducible of rank 3 or 4 . We will consider the totally exceptional double points for the Prym realization in 3 different cases. Each time, from the product structure around a totally exceptional pencil (as remarked at the end of 3.2) we can deduce the structure of the normal cone and its irreducibility.

6.3.1. Take as the base curve $C$ of genus 5 the normalization of a general plane quintic curve with a cusp. There is a base point free $g_{3}^{1}$ on $C$ cut by lines through the cusp point $p$, residually to the base locus $2 p$. Adding $p$ to the $g_{3}^{1}$ defines a semicanonical pencil since $\left|K_{C}\right|$ is cut by the conics through the cusp, residually to the base locus at the cusp. Thus $C$ is a trigonal curve with a "vanishing theta null" $M=\mathcal{O}_{C}\left(g_{3}^{1}+p\right)$. Consider any half-period $\eta$ on $C$ such that $h^{0}(M(\eta))=0$, and let $\pi: \widetilde{C} \rightarrow C$ be the resulting étale double cover. Then the Prym variety is a genus 4 Jacobian [Re, and $L=\pi^{*}(M)$ defines a vanishing theta null of $(P, \Xi)$; cf. [V] pp. 946-948]. Since $h^{0}(L)=h^{0}(M)+h^{0}(M(\eta))=2,|L|$ is a totally exceptional pencil, and the projective normal cone $\mathbb{P} C_{|L|}(X)$ parametrizes the projectivized tangent cone at the vanishing theta null point $L$, which is a rank 3 quadric surface containing the genus 4 canonical curve in $\mathbb{P}^{3}$. In particular, from the product structure we see that the threefold $\mathbb{P} C_{|L|}(X)$ is irreducible and rational and is singular along a line.

6.3.2. Take an even étale double cover $\widetilde{C}$ of a general plane quintic $C$, so that the Prym variety $(P, \Xi)$ is a general genus 5 Jacobian. Here "even" means that $h^{0}\left(\mathcal{O}_{C}\left(g_{5}^{2}\right)(\eta)\right)=0$ instead of 1 , as in the case when the Prym is the intermediate Jacobian of a cubic threefold. It is known that $\operatorname{Sing}(\Xi) \cong \widetilde{C}$ and the -1 map on $P$ induces the involution $\iota$ on $\widetilde{C}$, thus $\operatorname{Sing}(\Xi) /(-1) \cong C$. Explicitly, if $q$ is a point of the quintic curve $C \subset \mathbb{P}^{2}$, then the $g_{4}^{1}$ on $C$ swept out by lines through $q$ lifts to a $g_{8}^{1}$ on $\widetilde{C}$, and if we add twice one of the two preimages of $q$, say $w$, we get a point of $\operatorname{Sing}(\Xi)$.

In other words, consider $L=\pi^{*}\left(g_{4}^{1}\right)(2 w)$. First, the norm of $L$ is the canonical divisor class on $C$ since $2 g_{4}^{1}+2 q \equiv 2 g_{5}^{2} \equiv K_{C}$. Second, we claim that $h^{0}(L)=2$, so that $|L|=\pi^{*}\left|g_{4}^{1}\right|+2 w$ is a $g_{10}^{1}$ on $\widetilde{C}$ with base divisor $2 w$.

Proof. Note that $H^{0}\left(\pi^{*}\left(g_{4}^{1}\right)(w+\iota w)\right)=H^{0}\left(\pi^{*}\left(g_{5}^{2}\right)\right) \cong H^{0}\left(g_{5}^{2}\right) \oplus H^{0}\left(g_{5}^{2}(\eta)\right)=$ $H^{0}\left(g_{5}^{2}\right)$ is 3-dimensional, coming from the linear forms on the plane, so all of the linear series $\left|\pi^{*}\left(g_{4}^{1}\right)(w+\iota w)\right|$ on $\widetilde{C}$ is pulled back from $C$. But we can inject $\left|\pi^{*}\left(g_{4}^{1}\right)(w)\right| \hookrightarrow\left|\pi^{*}\left(g_{4}^{1}\right)(w+\iota w)\right|$ by adding $\iota w$, so we see that $\left|\pi^{*}\left(g_{4}^{1}\right)(w)\right|=\pi^{*}\left|g_{4}^{1}\right|+$ $w$, hence $h^{0}\left(\pi^{*}\left(g_{4}^{1}\right)(w)\right)=2$. Therefore $h^{0}\left(\pi^{*}\left(g_{4}^{1}\right)(2 w)\right) \leq 3$. But $h^{0}\left(\pi^{*}\left(g_{4}^{1}\right)(2 w)\right)$ and $h^{0}\left(\pi^{*}\left(g_{4}^{1}\right)(w+\iota w)\right)$ have opposite parity by Mumford's flipping property (cf. S-V6, p. 233], so $h^{0}\left(\pi^{*}\left(g_{4}^{1}\right)(2 w)\right)=2$ and $2 w$ is in the base locus (therefore equal to the base divisor since $\left|g_{4}^{1}\right|$ and hence $\left|\pi^{*}\left(g_{4}^{1}\right)\right|$ is base point free).

Thus $L$ is a totally exceptional singular point of $\Xi$ and the fiber $|L|$ is a $\mathbb{P}^{1}$. In this case the divisor variety $X$ is a $\mathbb{P}^{1}$ bundle over $\Xi$, and therefore the normal cone to $|L|$ in $X$ is $\mathbb{P}^{1}$ crossed with the tangent cone of $\Xi$ at $L$. Since $(P, \Xi)$ is a general genus 5 Jacobian all the singular points of $\Xi$ are rank 4 double points, so $C_{L}(\Xi)$ is a rank 4 quadric in the 5 -dimensional vector space $T_{L}(P)$. In particular the projective normal cone $\mathbb{P} C_{|L|}(X)$ is irreducible and rational, merely the product of $\mathbb{P}^{1}$ and a rank 4 quadric threefold in $\mathbb{P}^{4}$. 
6.3.3. Take a general trigonal curve $C$ of genus $g \geq 6$ and any connected étale double cover $\widetilde{C} \rightarrow C$. Then [Re the Prym variety $(P, \Xi)$ is the Jacobian of a genus $p=g-1$ tetragonal curve $Z$, which is also general, hence not trigonal and (when $p=6$ ) not a plane quintic. Therefore, by Petri's theorem the canonical model $Z \subset \mathbb{P}^{p-1}$ is cut out by quadrics, and by Green's theorem the space of quadrics containing the canonical curve is spanned by the projectivized tangent cones at double points of the theta divisor of the Jacobian of $Z$. It follows that for a general double point $L$ of $\Xi$, the projectivized tangent cone $\mathbb{P} C_{L}(\Xi)$ cannot contain the Prym canonical model of $C$ in $\mathbb{P}^{p-1}$, since the intersection of all these quadrics is $Z$, not $C$.

Thus $h^{0}(L)=2$ for general $L \in \operatorname{Sing}(\Xi)$ since if $h^{0}(L)=4$, then $L \in \Xi$ is a stable double point and the projective quadric tangent cone contains the Prym canonical curve; cf. S-V3, Prop. 5.1]. Therefore a general singular point $L$ of $\Xi$ is totally exceptional, and the fiber $|L| \subset X$ is a $\mathbb{P}^{1}$. Hence as before the projective normal cone $\mathbb{P} C_{|L|}(X)$ is the product of $\mathbb{P}^{1}$ and a rank 4 quadric threefold in $\mathbb{P}^{4}$.

We note that every point $L \in \operatorname{Sing}(\Xi)$ has the form $\pi^{*}\left(g_{3}^{1}\right)(B)$ for some effective divisor $B$ on $\widetilde{C}$ such that $N m(B) \in\left|K_{C}-2 g_{3}^{1}\right|$.

Proof. Note first that $h^{0}\left(2 g_{3}^{1}\right)=3$. Then $h^{0}\left(K_{C}-2 g_{3}^{1}\right)=(2 g-2-6)+1-g+$ $h^{0}\left(2 g_{3}^{1}\right)=g-4$, so $\left|K_{C}-2 g_{3}^{1}\right| \cong \mathbb{P}^{g-5}$ and its preimage $N m^{-1}\left|K_{C}-2 g_{3}^{1}\right|$ has pure dimension $g-5=p-4$. Now map $\pi^{*}\left|g_{3}^{1}\right| \times N m^{-1}\left|K_{C}-2 g_{3}^{1}\right|$ to $\widetilde{C}^{(2 g-2)}$. The image has pure dimension $p-3$ and lies in the locus $N m^{-1}\left|K_{C}\right|$, which consists of 2 disjoint irreducible components, according to the parity of $h^{0}(D)$ for $D \in \widetilde{C}^{(2 g-2)}$ with $N m(D) \in\left|K_{C}\right|$. Therefore, there is an irreducible variety $V \subset N m^{-1}\left|K_{C}-2 g_{3}^{1}\right|$ of dimension $p-4$ such that $\pi^{*}\left|g_{3}^{1}\right| \times V$ maps to $X$ and the image has dimension $p-3$. Now we appeal to Mumford's result [M, Lemma, p. 345] that, since $\operatorname{Sing}(\Xi)$ has dimension $p-4$, the general point of $\operatorname{Sing}(\Xi)$ is exceptional with respect to $\pi: \widetilde{C} \rightarrow C$. In other words, the only other form that a general point of $\operatorname{Sing}(\Xi)$ could have is for it to be $\pi^{*}\left(g_{4}^{1}\right)(B)$ where now $N m(B) \in\left|K_{C}-2 g_{4}^{1}\right|$. But for this possibility, $h^{0}\left(2 g_{4}^{1}\right) \leq 4$ by Clifford's theorem, hence $h^{0}\left(K_{C}-2 g_{4}^{1}\right) \leq(2 g-2-8)+$ $1-g+4=g-5$. Thus $\operatorname{dim}\left|K_{C}-2 g_{4}^{1}\right| \leq p-5$, so $\pi^{*}\left|g_{4}^{1}\right| \times N m^{-1}\left|K_{C}-2 g_{4}^{1}\right|$ and its image in $N m^{-1}\left|K_{C}\right|$ have dimension $\leq p-4$. Therefore, after intersecting with $X$ and mapping to $\Xi$, the image has dimension $\leq p-5$ and cannot contain a general point of $\operatorname{Sing}(\Xi)$.

6.4. Abel Prym differential for the trigonal Prym representations of a general genus 5 Jacobian. Continuing from above we consider in more detail the case that $g=6$, so $C$ is a general trigonal curve of genus 6 whose Prym variety $(P, \Xi)$ is the Jacobian of a general genus 5 curve $Z$.

An interesting new feature is that now $Z$ has a curve of $g_{4}^{1}$ 's, namely $\operatorname{Sing}(\Xi)$ itself, which is an étale double cover of the plane quintic discriminant curve in the net of all quadrics containing the canonical model of $Z$. As we showed, all the singularities of $\Xi$ have the form $L=\pi^{*}\left(g_{3}^{1}\right)(B)$ with $N m(B) \in\left|K_{C}-2 g_{3}^{1}\right|$ and $h^{0}(L)$ even. We want to determine exactly which of these double points $L$ are also stable. That is, when can we have $h^{0}(L)=4$ in this situation?

The claim is that if $\widetilde{\Delta} \rightarrow \Delta$ is the étale double cover of the plane quintic associated with $Z$, then there is a distinguished point $q \in \Delta$ such that for exactly the 2 points over $q$ in $\widetilde{\Delta} \cong \operatorname{Sing}(\Xi)$ the corresponding 2 points $L \in \operatorname{Sing}(\Xi)$ have 
$h^{0}(L)=4$ (with $L$ on $\widetilde{C}$, for $\pi: \widetilde{C} \rightarrow C$ with $C$ general genus 6 trigonal as described just above), and the rank 4 quadric threefold $Q \subset \mathbb{P}^{4}$ which contains $Z$ and is represented by $q$ also contains the Prym canonical model of $C$ (in the same $\mathbb{P}^{4}$ ). Among the quadrics in $\mathbb{P}^{p-1}$ precisely those that contain the Prym canonical curve come from $\operatorname{ker}\left(P^{*}\right)$, the kernel of the codifferential of the Prym map. According to [D-S. Prop. 3.3], this kernel has dimension 1 in the present situation. [We can give a slightly different argument as follows: by Sard's theorem applied to the preimage of the Jacobian locus along trigonals, with the smooth curve fibers, the image of the derivative $P_{*}$ contains the tangent space to the Jacobians at $Z$; hence the quadrics from $\operatorname{ker}\left(P^{*}\right)$ must contain the canonical curve $Z \subset \mathbb{P}^{p-1}$. However, if a quadric contains both $Z$ and the Prym canonical curve, it must be the quadric $Q$ that is swept out by the $g_{4}^{1}$. Namely, in each plane it has to contain the 4 points of $Z$ and the 3 intersection points (on $C$ ) of the line pairs, hence all 6 lines and hence the whole plane, so it must be $Q$.] The conclusion is that except for one conjugate pair of stable points $L$ on the curve $\operatorname{Sing}(\Xi)$, all the other singular points are totally exceptional with $h^{0}(L)=2$.

When $L$ is one of the two stable rank 4 double points, what is the structure of the parametrizing map $\mathbb{P} C_{|L|}(X) \rightarrow \mathbb{P} C_{L}(\Xi)=Q \subset \mathbb{P}^{4}$ ? Case 2) of Theorem 1 applies since $r k(Q)=4$. The projectivized normal cone $\mathbb{P} C_{|L|}(X)$ is irreducible, 4-dimensional, mapping onto $|L| \cong \mathbb{P}^{3}$ which contains a single isotropic $\mathbb{P}^{1}$ of the form $\pi^{*}\left|g_{3}^{1}\right|+B$. Over a point $D$ of $|L|$, the fiber is a $\mathbb{P}^{1}$ if $D$ is not on the isotropic $\mathbb{P}^{1}$ and is $\mathbb{P}^{2}$ if $D$ is on the isotropic $\mathbb{P}^{1}$. Thus the parametrizing map sweeps out $Q$ by an $\infty^{3}$ family of $\mathbb{P}^{1}$ 's and the generic fiber is a $\mathbb{P}^{1}$, but the restriction of the map to the 3 -dimensional subvariety lying over the isotropic $\mathbb{P}^{1}$ sweeps out $Q$ birationally by an $\infty^{1}$ family of $\mathbb{P}^{2}$ 's.

Finally, we note incidentally that for the Jacobian of a generic genus 5 curve $Z$, each Prym representation by an étale double cover of a smooth genus 6 curve $C$ is characterized almost completely by the associated set of stable double points on the Jacobian theta divisor $\Theta(Z)$. Recall from [D-S, p. 35 and sections II-III] that these Prym representations (with $C$ smooth) can occur in 2 ways: (1) a single one, of the form $\widetilde{\Delta}=\operatorname{Sing}(\Theta(Z)) \rightarrow \Delta$ where $\Delta$ is the plane quintic discriminant curve of $Z$ as in 6.3 .2 , and (2) a curve of them parametrized by $w \in \widetilde{\Delta}$, of the form $\widetilde{C} \rightarrow C$ with $C$ a trigonal curve as discussed here. In the singleton case (1) there are no stable double points of $\operatorname{Sing}(\Theta(Z))$, and in the curve case (2) if the double cover is indexed by $w \in \widetilde{\Delta}$, then the stable double points are the two conjugate points $\left\{w, w^{\prime}\right\}$ of $\operatorname{Sing}(\Theta(Z))$ lying over the same point $q$ of $\Delta$.

\section{A DEgEnERAte EXAMPle}

In this section we show by example that for an exceptional, but not very exceptional, singular point $L$ of a Prym theta divisor $\Xi$ the unique irreducible component of the normal cone $C_{|L|}(X)$ of the divisor variety $X$ that dominates $|L|$ need not always map onto an irreducible component of the tangent cone $C_{L}(\Xi)$. In particular, the normal cone $C_{|L|}(X)$ can be reducible in case (1) of section 3.1.

First we describe the simplest possible skew symmetric matrix of linear forms that would lead to this behavior. Then we show that this matrix can occur; the example is constructed from a rather special kind of trigonal genus 6 smooth curve $C$ and étale double cover. 
Proposition 1. Let $\pi: \widetilde{C} \rightarrow C$ be a connected étale double cover of a nonhyperelliptic smooth projective curve $C$ of genus $g$. Consider the divisor variety $X \subset \widetilde{C}^{(2 g-2)}$ and the parametrization $\varphi: X \rightarrow \Xi$ of the Prym theta divisor $\Xi \subset P$. Assume that $L \in \Xi$ is a point with $h^{0}(\widetilde{C}, L)=4$ for which Mumford's skew symmetric matrix of linear forms can be expressed as:

$$
\left(\begin{array}{cccc}
0 & 0 & x & y \\
0 & 0 & y & z \\
-x & -y & 0 & 0 \\
-y & -z & 0 & 0
\end{array}\right) \text {, with } x, y, z \text { linearly independent on } T_{L}(P)
$$

Then $L$ is an exceptional singular point of $\Xi$ and the following properties hold.

(a) The tangent cone $C_{L}(\Xi)$ has Pfaffian equation $x z-y^{2}=0$ and $L$ is a rank 3 double point on $\Xi$ at which $R S T$ holds. In particular, $L$ is not a very exceptional singular point.

(b) The normal cone $C_{|L|}(X)$ has exactly two irreducible components: $\mathcal{C}_{1}$ supported over all of $|L| \cong \mathbb{P}^{3}$ and $\mathcal{C}_{2}$ supported over a smooth quadric surface $Q \subset \mathbb{P}^{3}$. Now consider the mapping from each of these two p-dimensional varieties $\mathcal{C}_{1}, \mathcal{C}_{2}$ to $C_{L}(\Xi) \subset T_{L}(P) \cong \mathbb{C}^{p}$ induced by restriction of the surjective morphism $C_{|L|}(X) \rightarrow$ $C_{L}(\Xi)$. (We have set $p=g-1$ as usual.)

(1) $\mathcal{C}_{1} \subset|L| \times \mathbb{C}^{p}$ is equal to the product of $|L|$ and a codimension 3 vector subspace $E \subset \mathbb{C}^{p}$, so that the map from $\mathcal{C}_{1}$ to $\mathbb{C}^{p}$ is projection to the 2 nd factor of the product. In particular, the image in $T_{L}(P)$ of the injections from the fibers in $\mathcal{C}_{1}$ over the points $D \in|L|$ does not vary with $D$.

(2) The map from $\mathcal{C}_{2}$ is onto $C_{L}(\Xi)$.

Proof. First, $L$ is an exceptional singular point by [S-V5, Lemma 2.2 (ii), p. 450]. More precisely, if $\left\{s_{1}, \ldots, s_{4}\right\}$ is any basis for $H^{0}(\widetilde{C}, L)$ with respect to which the skew-symmetric $4 \times 4$ matrix of linear forms has the form displayed above in the assumption, then the span of the 1st two basis vectors $\left\{s_{1}, s_{2}\right\}$ is visibly an isotropic subspace $W$ for $\beta$, and so there is a line bundle $M$ on $C$ and a vector subspace $\Lambda \subset H^{0}(C, M)$ such that $L \cong \pi^{*}(M)(\mathcal{B})$, for some divisor $\mathcal{B} \geq 0$ on $\widetilde{C}$, and $W=\pi^{*}(\Lambda) \cdot u$ where $\operatorname{div}(u)=\mathcal{B}$. Thus $h^{0}(M) \geq 2$ and $L$ is an exceptional singularity of $\Xi$. (In fact, $W \subset H^{0}(L)$ is a maximal isotropic subspace for $\beta$ since the linear forms $x, y$ in the first row are linearly independent and $y, z$ in the second row are linearly independent. Thus $h^{0}(M)=2$; cf. [S-V5, Lemma 2.3 (ii), p. 451]. Similarly, for the span $W_{2}$ of the last two basis vectors $\left\{s_{3}, s_{4}\right\}$, there is another line bundle $M_{2}$ on $C$ with $h^{0}\left(M_{2}\right)=2$ and $L \cong \pi^{*}\left(M_{2}\right)\left(\mathcal{B}_{2}\right), W_{2}=\pi^{*}\left(H^{0}\left(C, M_{2}\right)\right) \cdot u_{2}$; however, in the present situation, case 3) (ii) of Theorem 1, there are $\infty^{1}$ such exceptional realizations of $L$.)

Next, the conclusions stated in part (a) are immediate. Namely, the Pfaffian of the skew-symmetric $4 \times 4$ matrix is $x z-y^{2}$, which is a rank 3 quadratic form on $T_{L}(P) \cong \mathbb{C}^{p}$. In particular, since the Pfaffian polynomial is not identically zero, it is an equation for the tangent cone $C_{L}(\Xi)$, RST holds, and $L$ is not a very exceptional singularity of $\Xi$.

Finally, everything in part (b) will follow by direct computation with the matrix of linear forms. With respect to the basis for $H^{0}(L)$, consider the point 
$D=(a, b, c, d) \in \mathbb{P}^{3} \cong|L|$. Then the 4 linear forms appearing in the column vector

$$
\left(\begin{array}{cccc}
0 & 0 & x & y \\
0 & 0 & y & z \\
-x & -y & 0 & 0 \\
-y & -z & 0 & 0
\end{array}\right) \cdot\left(\begin{array}{l}
a \\
b \\
c \\
d
\end{array}\right)=\left(\begin{array}{c}
c x+d y \\
c y+d z \\
-a x-b y \\
-a y-b z
\end{array}\right)
$$

define in $T_{L}(P) \cong T_{0}(P) \cong \mathbb{C}^{p}$ the image subspace $\varphi_{*}\left(T_{D}(X)\right)$ of the Abel Prym differential at $D$.

The span of the linear forms in this column vector is not all of $\mathbb{C} x+\mathbb{C} y+\mathbb{C} z$ if and only if $a d-b c=0$. Indeed, expressing the 4 linear forms in terms of the linearly independent ones $x, y, z$ gives the matrix $\left(\begin{array}{ccc}c & d & 0 \\ 0 & c & d \\ -a & -b & 0 \\ 0 & -a & -b\end{array}\right)$, and the rank of this $4 \times 3$ matrix is $<3$ iff $a d-b c=0$.

Let $\mathcal{C}_{1}$ be the irreducible component of the normal cone $C_{|L|}(\Xi)$ that dominates $|L|$. Since for $a d-b c \neq 0$, the image $\varphi_{*}\left(T_{D}(X)\right) \subset T_{L}(P)$ is constantly the codimension 3 subspace $E$ defined by $x, y, z$, this component $\mathcal{C}_{1}$ is $|L| \times E$ and does not map onto the irreducible tangent cone $C_{L}(\Xi)$. Therefore, there must be another irreducible component of the normal cone that maps onto $C_{L}(\Xi)$.

Now when the point $D$ of $|L| \cong \mathbb{P}^{3}$ lies on the smooth quadric surface $Q: a d-b c=$ 0 , the 4 linear forms span only a 2-dimensional vector space of linear forms, so $\varphi_{*}\left(T_{D}(X)\right) \subset T_{L}(P)$ has codimension 2 in the p-dimensional vector space $T_{L}(P)$. Thus over $Q$ the fibers of the normal space $N_{|L|}(X)$ are all (p-2)-dimensional, so there is an irreducible p-dimensional component $\mathcal{C}_{2}$ of $N_{|L|}(X)$ lying over and dominating $Q \subset|L|$ (forming a rank p-2 vector bundle over $Q$ ). Since we know that there is at least one irreducible component of $C_{|L|}(X)$ besides $\mathcal{C}_{1}$, and $\mathcal{C}_{2}$ accounts for all of $N_{|L|}(X)$ residually to $\mathcal{C}_{1}$, then $\mathcal{C}_{2}$ must be an irreducible component of the normal cone and the only one besides $\mathcal{C}_{1}$.

Having proved the statements we made about the general structure of $\mathcal{C}_{2}$ with its surjective map to $C_{L}(\Xi)$, we note incidentally the following explicit structure of the map. If we express a point $(a, b, c, d) \in Q$ (the rank 4 quadric surface $a d-b c=0$ in $\left.\mathbb{P}^{3} \cong|L|\right)$ as $\left(\lambda_{0} \mu_{0}, \lambda_{0} \mu_{1}, \lambda_{1} \mu_{0}, \lambda_{1} \mu_{1}\right)$ for $\left(\lambda_{0}, \lambda_{1}\right),\left(\mu_{0}, \mu_{1}\right) \in \mathbb{P}^{1}$, then the projection map $\mathcal{C}_{2} \subset|L| \times T_{L}(P) \rightarrow T_{L}(P)$ simply sends the (p-2)-dimensional vector space fiber in $\mathcal{C}_{2}$ over $\left(\lambda_{0} \mu_{0}, \lambda_{0} \mu_{1}, \lambda_{1} \mu_{0}, \lambda_{1} \mu_{1}\right)$ to the codimension 2 subspace of $T_{L}(P) \cong \mathbb{C}^{p}$ defined by $\mu_{0} x+\mu_{1} y$ and $\mu_{0} y+\mu_{1} z$.

Proposition 2. Assume that $C$ is a connected nonhyperelliptic smooth projective curve of genus $g$ with 2 vanishing theta nulls $M_{1}, M_{2}$ of the following particular form: $M_{1} \cong M_{0}\left(B_{1}\right), M_{2} \cong M_{0}\left(B_{2}\right)$, for effective divisors $B_{1}, B_{2}$ and a line bundle $M_{0}$ such that $\left|M_{0}\right|$ is a base point free pencil, $\left|M_{1}\right|=\left|M_{0}\right|+B_{1},\left|M_{2}\right|=\left|M_{0}\right|+B_{2}$, and $\eta=B_{1}-B_{2}$ is a nontrivial half-period. In other words, $\left|M_{1}\right|$ and $\left|M_{2}\right|$ are distinct vanishing theta null pencils on $C$ with the same moving part $\left|M_{0}\right|$ and distinct base divisors $B_{1}$ and $B_{2}$.

Let $\pi: \widetilde{C} \rightarrow C$ be the étale double cover of $C$ defined by $\eta=B_{1}-B_{2}$, and consider $L=\pi^{*}\left(M_{1}\right)$ on $\widetilde{C}$. Then $L \in \Xi$ has $h^{0}(\widetilde{C}, L)=4$, and Mumford's skew symmetric matrix of linear forms can be expressed in the form displayed in Proposition 1.

Proof. Since $M_{1} \cong M_{2}(\eta)$ on $C$ and $\pi^{*}(\eta)=0$ on $\widetilde{C}$ by construction, $\pi^{*}\left(M_{1}\right) \cong$ $\pi^{*}\left(M_{2}\right)$. Therefore, by the projection formula, $H^{0}(L) \cong H^{0}\left(M_{1}\right) \oplus H^{0}\left(M_{2}\right)$ has 
dimension $2+2=4$. (In detail, $H^{0}(L) \cong H^{0}\left(\pi^{*}\left(M_{1}\right)\right) \cong H^{0}\left(\pi_{*} \pi^{*}\left(M_{1}\right)\right) \cong$ $H^{0}\left(M_{1} \otimes \pi_{*}\left(\mathcal{O}_{\widetilde{C}}\right)\right) \cong H^{0}\left(M_{1} \otimes\left(\mathcal{O}_{C} \oplus \mathcal{O}_{C}(\eta)\right) \cong H^{0}\left(M_{1}\right) \oplus H^{0}\left(M_{2}\right)\right.$.)

Now the determination of the skew symmetric $4 \times 4$ matrix of linear forms is just like [S-V3, Ex. 2.18, p. 492] and [S-V4, Prop. 3.6, p. 246], except that everything has been pre-arranged here so that the rank of the Pfaffian quadratic form is 3 , not 4. Namely, the upper right $2 \times 2$ block $\Lambda$ of linear forms comes from the cup product $H^{0}\left(M_{1}\right) \times H^{0}\left(M_{1}(\eta)\right) \rightarrow H^{0}\left(\Omega_{C}(\eta)\right)$. If the two base divisors $B_{1}$ and $B_{2}$, for $\left|M_{1}\right|$ and $\left|M_{2}\right|=\left|M_{1}(\eta)\right|$ resp., are defined by sections $\beta_{i}$ of $\mathcal{O}_{C}\left(B_{i}\right), i=1,2$, then the section $\beta_{1} \beta_{2}$ of $\mathcal{O}_{C}\left(B_{1}+B_{2}\right)$ is a common factor of all the entries of $\Lambda$. Dividing out $\beta_{1} \beta_{2}$, we have the $2 \times 2$ block of linear forms coming from the cup product $H^{0}\left(M_{0}\right) \times H^{0}\left(M_{0}\right) \rightarrow H^{0}\left(M_{0}^{2}\right)$. Now for any pencil $\left|M_{0}\right|$, the linear map $H^{0}\left(M_{0}\right) \otimes_{\mathbb{C}} H^{0}\left(M_{0}\right) \rightarrow H^{0}\left(M_{0}^{2}\right)$ has rank exactly 3 since projectively the morphism $\mathbb{P}^{1} \times \mathbb{P}^{1} \rightarrow \mathbb{P} H^{0}\left(M_{0}^{2}\right)$ given by addition of divisors in the pencil has finite fibers. Finally, if $s, t$ are a basis for $H^{0}\left(M_{0}\right)$, then the matrix of cup products is $\left(\begin{array}{cc}s^{2} & s t \\ t s & t^{2}\end{array}\right)=\left(\begin{array}{ll}s^{2} & s t \\ s t & t^{2}\end{array}\right)$. Thus $\Lambda=\left(\begin{array}{cc}s^{2} & s t \\ s t & t^{2}\end{array}\right) \cdot \beta_{1} \beta_{2}=\left(\begin{array}{ll}x & y \\ y & z\end{array}\right)$, as desired.

Proposition 3. There exists a (nonhyperelliptic) trigonal connected smooth projective curve $C$ of genus 6 with 2 vanishing theta nulls $M_{1}, M_{2}$ that satisfy all the properties postulated in Proposition 2.

Proof. We will construct $C$ as the normalization of a degree 6 plane curve with certain singularities and special tangent lines. First, two lemmas.

Lemma 1. Let $Y \subset \mathbb{P}^{2}$ be an irreducible sextic curve with a triple point $P$ and a double point $Q$, both ordinary, and no other singularities. Let $C \rightarrow Y$ be the normalization. Then $C$ is a connected smooth projective curve of genus 6 and is trigonal, nonhyperelliptic. Moreover, for any line $\ell$ through $Q$ that is tangent to $Y$ at 2 smooth points $a$ and $b$, there is an associated vanishing theta null pencil on $C$ with base divisor $a+b$.

Proof. $C$ is a smooth projective curve by construction, since the normalization of any projective curve is a smooth projective curve. $C$ is irreducible since $Y$ is; hence $C$ is connected. Now we compute the genus of $C$. At an ordinary plane curve singularity of multiplicity $m$, the "diminution of the genus" is given by the formula $\delta=\frac{m(m-1)}{2}$. Thus $\delta_{P}=3$ and $\delta_{Q}=1$, and hence $g(C)=10-(3+1)=6$ (since $Y$ has arithmetic genus $\left.p_{a}(Y)=(6-1)(6-2) / 2=10\right)$.

The pencil of lines through $P$ in the plane cut a moving degree 3 linear series on $Y$. More precisely, if $p_{1}, p_{2}, p_{3}$ are the 3 points of $C$ mapping to $P$ in $Y$ (the "branches" of $Y$ at $P$ ), then $\left|\mathcal{O}_{C}(1)\left(-p_{1}-p_{2}-p_{3}\right)\right|$ is a base point free pencil of degree 3 on $C$, where $\mathcal{O}_{C}(1)$ on $C$ is the pullback of the line bundle $\mathcal{O}_{Y}(1)$ on $Y$. Since $C$ admits a $g_{3}^{1}$ (degree 3 pencil), $C$ is trigonal; and $C$ is nonhyperelliptic since this $g_{3}^{1}$ is base point free (and $g>2$ ).

Now assume that $\ell \subset \mathbb{P}^{2}$ is a line through $Q$ that is bitangent to $Y$, at distinct smooth points $a$ and $b$. Set $B=a+b$ on $C$, and let $G$ denote the $g_{3}^{1}$ line bundle $\mathcal{O}_{C}(1)\left(-p_{1}-p_{2}-p_{3}\right)$. Then consider the degree 5 line bundle $M=G(B)$ on $C$. We must show that $M^{\otimes 2} \cong \Omega_{C}^{1}, h^{0}(M)=2$, and $|M|=|G|+B\left(=\left|g_{3}^{1}\right|+B\right.$, the $g_{3}^{1}$ pencil plus base divisor $B$ ).

On one hand, $M^{\otimes 2} \cong \mathcal{O}_{C}(2)\left(-2 p_{1}-2 p_{2}-2 p_{3}\right)(2 a+2 b)$, and using the line $\overline{P Q}$ to get the divisor $2\left(p_{1}+p_{2}+p_{3}+q_{1}+q_{2}+r\right)$ in $\left|\mathcal{O}_{C}(2)\right|$, we can represent $M^{\otimes 2}$ 
by the divisor class $2\left(p_{1}+p_{2}+p_{3}+q_{1}+q_{2}+r\right)-\left(2 p_{1}+2 p_{2}+2 p_{3}\right)+(2 a+2 b)=$ $2 q_{1}+2 q_{2}+2 r+2 a+2 b$. On the other hand, the canonical series $\left|\Omega_{C}^{1}\right|$ is cut by the special adjoints, i.e. the plane cubics which are singular at $P$ and contain $Q$, residual to the assigned base divisor $2 p_{1}+2 p_{2}+2 p_{3}+q_{1}+q_{2}$, where $q_{1}, q_{2}$ are the 2 points of $C$ mapping to $Q$ in $Y$ (the "branches" of $Y$ at $Q$ ). (In particular, the degree checks: $3 \cdot 6-8=10$.) Thus take for an adjoint cubic the line $\ell$ plus twice the line through $P$ and $Q$. The divisor on $C$ that this cubic curve cuts is $\left(q_{1}+q_{2}+2 a+2 b\right)+2\left(p_{1}+p_{2}+p_{3}+q_{1}+q_{2}+r\right)$, where $r$ is the 6 th point of intersection of $\overline{P Q}$ with $Y$. Subtracting the assigned base divisor gives the canonical divisor: $\left(2\left(p_{1}+p_{2}+p_{3}\right)+3\left(q_{1}+q_{2}\right)+2 r+2 a+2 b\right)-\left(2 p_{1}+2 p_{2}+2 p_{3}+q_{1}+q_{2}\right)=$ $2 q_{1}+2 q_{2}+2 r+2 a+2 b$. Thus, $M^{\otimes 2} \cong \Omega_{C}^{1}$.

To show the rest, since $h^{0}(G)=2$ and $|G|+B \subset|M|$, it will suffice to check that $h^{0}(M)<3$. By Clifford's theorem, $h^{0}(M) \leq 3$. So consider the possibility that $h^{0}(M)=3$. Then the net $|M|$ must be base point free, since otherwise, by subtracting a base point we could get a $g_{4}^{2}$ and $C$ would be hyperelliptic, a contradiction. Thus $|M|$ would have to be a base point free $g_{5}^{2}$ and map $C$ onto a plane quintic curve. This map would have to be an isomorphism from $C$ to a smooth plane quintic, since a plane quintic has arithmetic genus 6 and $C$ is smooth of genus 6. However, a smooth plane quintic curve is never trigonal (since any degree 3 effective divisor $D$ on a smooth plane quintic imposes 3 independent linear conditions for plane conics to cut a divisor $\geq D$, so $D$ cannot move in a linear series).

Lemma 2. There exists an irreducible sextic curve $Y \subset \mathbb{P}^{2}$ with a triple point $P$ and a double point $Q$, both ordinary, and no other singularities, with the property that there are 2 distinct lines through $Q$ each of which is tangent to $Y$ at 2 smooth points.

Proof. We will work in $\mathbb{P}^{2}$ with a point $\mathrm{Q}, 2$ distinct lines through $\mathrm{Q}$, the proposed points of bitangency, a point $\mathrm{P}$ not on the 2 lines, and apply Bertini's theorem to get the existence of $Y$. Let's begin in the affine plane with $Q=(0,0)$, the $x$-axis and the $y$-axis, points $\left(a_{1}, 0\right),\left(b_{1}, 0\right)$ on the $x$-axis and points $\left(0, a_{2}\right),\left(0, b_{2}\right)$ on the $y$-axis, and the point $P=(c, c)$. Now consider the affine plane sextics with the following types of equations: $A=\left(x \alpha_{1} \beta_{1}\right)^{2}, B=\left(y \alpha_{2} \beta_{2}\right)^{2}$, and $\mathcal{C}=x y \cdot\{h(x, y)\}$, where $\alpha_{1}$ is an affine equation for the line through $\left(a_{1}, 0\right)$ and $(c, c), \beta_{1}$ is an affine equation for the line through $\left(b_{1}, 0\right)$ and $(c, c)$ (similarly for $\left.\alpha_{2}, \beta_{2}\right)$, and $\{h(x, y)\}$ are the affine quartics with at least a triple point at $(c, c)$. Now homogenize and consider the linear system generated by $A, B$ and $\mathcal{C}$. Then there are no base points in $\mathbb{P}^{2}$ outside the assigned ones $\left(\left(a_{1}, 0\right),\left(b_{1}, 0\right),\left(0, a_{2}\right),\left(0, b_{2}\right), Q=(0,0)\right.$, and $P=(c, c)$ in the affine plane), and all members $Y$ of this linear system of sextic curves in $\mathbb{P}^{2}$ have the property that $P$ is a singular point of multiplicity $\geq 3, Q$ is a singular point of multiplicity $\geq 2$, and the 2 affine coordinate axes are tangent to $Y$ at $\left(a_{1}, 0\right)$ and $\left(b_{1}, 0\right),\left(0, a_{2}\right)$ and $\left(0, b_{2}\right)$. For the local conditions: that $P$ is an ordinary triple point, that $Q$ is an ordinary double point, and that $\left(a_{1}, 0\right),\left(b_{1}, 0\right),\left(0, a_{2}\right),\left(0, b_{2}\right)$ are all smooth points, it is clear that there are members satisfying these conditions (namely, general $x y h \in \mathcal{C}$ ), and hence that the general member satisfies all of these local conditions. Then Bertini does the rest: irreducible and no unassigned singularities. 
Now we complete the proof of Proposition 3. Take an irreducible plane sextic curve $Y$ as in Lemma 2, and let $C$ be the normalization, which is trigonal as in Lemma 1. Let $M_{0}$ be the $g_{3}^{1}$ line bundle (denoted by $G$ in the proof of Lemma 1), so that $\left|M_{0}\right|$ is a base point free pencil on $C$. Then, if the 2 bitangent lines through $Q$ are tangent to $Y$ at smooth points $a_{1}, b_{1}$ and $a_{2}, b_{2}$ resp., set $B_{1}=a_{1}+b_{1}$ and $B_{2}=a_{2}+b_{2}$ on $C$, and finally let $M_{i}=M_{0}\left(B_{i}\right)$ for $i=1,2$. By Lemma 1, each $\left|M_{i}\right|=\left|M_{0}\right|+B_{i}$ is a vanishing theta null pencil with base divisor $B_{i}$. The only thing that remains to be checked is that the half-period $\eta=B_{1}-B_{2}$ is nontrivial. By construction, $B_{1}$ and $B_{2}$ are distinct effective divisors of degree 2 on $C$. Since $C$ is nonhyperelliptic, $B_{1}$ and $B_{2}$ cannot be linearly equivalent.

Corollary (of Propositions 1-3). There exists a genus 6 smooth trigonal curve $C$ with an étale double cover $\pi: \widetilde{C} \rightarrow C$ for which the theta divisor $\Xi$ of the Prym variety has an exceptional, but not very exceptional, singular point $L$ such that the surjective map

$$
C_{|L|}(X) \rightarrow C_{L}(\Xi)
$$

from the normal cone $C_{|L|}(X)$ of the divisor variety $X$ along $|L|$, to the tangent cone $C_{L}(\Xi)$ of the Prym theta divisor at $L$, has the following structure. The tangent cone $C_{L}(\Xi)$ is irreducible and is parametrized by the part of $C_{|L|}(X)$ supported over $|L| \cap \operatorname{Sing}(X)$, not by the unique irreducible component that dominates $|L|$. In particular, the normal cone $C_{|L|}(X)$ is reducible.

Remark. The Prym variety appearing in the Corollary is a nonhyperelliptic genus 5 Jacobian (by results of Mumford and Recillas; see [M], Re, and also [B1, Thm. 4.10, p. 170]), and the rank 3 double point $L$ on the theta divisor is a vanishing theta null.

\section{ACKNOWLEDGEMENT}

The authors are grateful to the referee for helpful, detailed suggestions on improving the presentation of the contents.

\section{REFERENCES}

[A-M] A. Andreotti and A. L. Mayer, On period relations for abelian integrals on algebraic curves, Ann. Scuola Norm. Sup. Pisa (3) 21 (1967), 189-238. MR0220740 (36 \#3792)

[ACGH] E. Arbarello, M. Cornalba, P. A. Griffiths, and J. Harris, Geometry of algebraic curves. Vol. I, Grundlehren der Mathematischen Wissenschaften [Fundamental Principles of Mathematical Sciences], vol. 267, Springer-Verlag, New York, 1985. MR770932 (86h:14019)

[B1] Arnaud Beauville, Prym varieties and the Schottky problem, Invent. Math. 41 (1977), no. 2, 149-196. MR0572974 (58 \#27995)

[B2] Arnaud Beauville, Variétés de Prym et jacobiennes intermédiaires (French), Ann. Sci. École Norm. Sup. (4) 10 (1977), no. 3, 309-391. MR0472843 (57 \#12532)

[B3] Arnaud Beauville, Les singularités du diviseur $\Theta$ de la jacobienne intermédiaire de l'hypersurface cubique dans $\mathbf{P}^{4}$ (French), Algebraic threefolds (Varenna, 1981), Lecture Notes in Math., vol. 947, Springer, Berlin-New York, 1982, pp. 190-208. MR672617 (84c:14030)

[Bo] Armand Borel, Linear algebraic groups, Notes taken by Hyman Bass, W. A. Benjamin, Inc., New York-Amsterdam, 1969. MR0251042 (40 \#4273)

[CM] Sebastian Casalaina-Martin, Singularities of the Prym theta divisor, Ann. of Math. (2) 170 (2009), no. 1, 162-204, DOI 10.4007/annals.2009.170.163. MR2521114|(2010i:14052)

[D] Olivier Debarre, Sur le probleme de Torelli pour les varieties de Prym (French), Amer. J. Math. 111 (1989), no. 1, 111-134, DOI 10.2307/2374482. MR980302 (90b:14035) 
[D-S] Ron Donagi and Roy Campbell Smith, The structure of the Prym map, Acta Math. 146 (1981), no. 1-2, 25-102, DOI 10.1007/BF02392458. MR594627 (82k:14030b)

[F] William Fulton, Intersection theory, Ergebnisse der Mathematik und ihrer Grenzgebiete (3) [Results in Mathematics and Related Areas (3)], vol. 2, Springer-Verlag, Berlin, 1984. MR:732620 (85k:14004)

[G] M. L. Green, Quadrics of rank four in the ideal of a canonical curve, Invent. Math. 75 (1984), no. 1, 85-104, DOI 10.1007/BF01403092. MR728141 (85f:14028)

[H] Joe Harris, Algebraic geometry: A first course, Graduate Texts in Mathematics, vol. 133, Springer-Verlag, New York, 1992. MR1182558 (93j:14001)

[K1] George Kempf, On the geometry of a theorem of Riemann, Ann. of Math. (2) 98 (1973), 178-185. MR0349687 (50 \#2180)

[K2] G. Kempf, Topics on Riemann surfaces, Univ. Nac. Aut. Mexico, 1973, typed lecture notes, $27 \mathrm{pp}$.

[K3] George Kempf, Abelian integrals, Monografías del Instituto de Matemáticas [Monographs of the Institute of Mathematics], vol. 13, Universidad Nacional Autónoma de México, México, 1983. MR743421 (85j:14082)

[I-L] E. Izadi and H. Lange, Counter-examples of high Clifford index to Prym-Torelli, J. Algebraic Geom. 21 (2012), no. 4, 769-787, DOI 10.1090/S1056-3911-2012-00587-6. MR 2957696

[M-M] A. Mattuck and A. Mayer, The Riemann-Roch theorem for algebraic curves, Ann. Scuola Norm. Sup. Pisa (3) 17 (1963), 223-237. MR0162798 (29 \#102)

[M] David Mumford, Prym varieties. I, Contributions to analysis (a collection of papers dedicated to Lipman Bers), Academic Press, New York, 1974, pp. 325-350. MR0379510 (52 \#415)

[Re] Sevin Recillas, Jacobians of curves with $g_{4}^{1}$ 's are the Prym's of trigonal curves, Bol. Soc. Mat. Mexicana (2) 19 (1974), no. 1, 9-13. MR0480505 (58 \#666)

[Ri] Bernhard Riemann, Collected papers, Translated from the 1892 German edition by Roger Baker, Charles Christenson and Henry Orde, Kendrick Press, Heber City, UT, 2004. MR:2121437(2005m:01028)

[Shaf] Igor R. Shafarevich, Basic algebraic geometry. 1: Varieties in projective space, 2nd ed., Translated from the 1988 Russian edition and with notes by Miles Reid, Springer-Verlag, Berlin, 1994. MR1328833 (95m:14001)

[Shok] V. V. Shokurov, Prym varieties: Theory and applications (Russian), Izv. Akad. Nauk SSSR Ser. Mat. 47 (1983), no. 4, 785-855; English transl., Math. USSR-Izv. 23 (1984), 83-147. MR712095(85e:14040)

[S-V1] Roy Smith and Robert Varley, Tangent cones to discriminant loci for families of hypersurfaces, Trans. Amer. Math. Soc. 307 (1988), no. 2, 647-674, DOI 10.2307/2001192. MR.940221 (89e:32029)

[S-V2] Roy Smith and Robert Varley, Singularity theory applied to $\Theta$-divisors, Algebraic geometry (Chicago, IL, 1989), Lecture Notes in Math., vol. 1479, Springer, Berlin, 1991, pp. 238-257, DOI 10.1007/BFb0086273. MR1181216(93i:14042)

[S-V3] Roy Smith and Robert Varley, A Riemann singularities theorem for Prym theta divisors, with applications, Pacific J. Math. 201 (2001), no. 2, 479-509, DOI 10.2140/pjm.2001.201.479. MR.1875904 (2003d:14039)

[S-V4] R. Smith and R. Varley, The Prym Torelli problem: an update and a reformulation as a question in birational geometry, Symposium in Honor of C. H. Clemens (Salt Lake City, UT, 2000), Contemp. Math., vol. 312, Amer. Math. Soc., Providence, RI, 2002, pp. 235-264, DOI 10.1090/conm/312/05390. MR1941584(2003j:14011)

[S-V5] Roy Smith and Robert Varley, A necessary and sufficient condition for Riemann's singularity theorem to hold on a Prym theta divisor, Compos. Math. 140 (2004), no. 2, 447-458, DOI 10.1112/S0010437X03000320. MR2027198 (2005b:14059)

[S-V6] Roy Smith and Robert Varley, The Pfaffian structure defining a Prym theta divisor, The geometry of Riemann surfaces and abelian varieties, Contemp. Math., vol. 397, Amer. Math. Soc., Providence, RI, 2006, pp. 215-236, DOI 10.1090/conm/397/07475. MR2218011 (2007g:14034) 
[T] A. N. Tjurin, The geometry of the Poincaré divisor of a Prym variety (Russian), Izv. Akad. Nauk SSSR Ser. Mat. 39 (1975), no. 5, 1003-1043; English transl., Math. USSRIzv. 9 (1975), no. 5, 951-986. Corrections, Izv. Akad. Nauk SSSR Ser. Mat. 42 (1978), no. 2, 468; English transl., Math. USSR-Izv. 12 (1978), 438. MR0414563

[V] Robert Varley, Weddle's surfaces, Humbert's curves, and a certain 4-dimensional abelian variety, Amer. J. Math. 108 (1986), no. 4, 931-952, DOI 10.2307/2374519. MR853219 (87g:14050)

[W] Gerald E. Welters, A theorem of Gieseker-Petri type for Prym varieties, Ann. Sci. École Norm. Sup. (4) 18 (1985), no. 4, 671-683. MR839690 (88a:14034)

Department of Mathematics, University of Georgia, Athens, Georgia 30602

E-mail address: roy@math.uga.edu

Department of Mathematics, University of Georgia, Athens, Georgia 30602

E-mail address: rvarley@math.uga.edu 